\title{
Looking at Large Data Sets Using Binned Data Plots
}

D. B. Carr

April 1990

Prepared for the U.S. Department of Energy under Contract DE-AC06-76RLO 1830

Pacific Northwest Laboratory

Operated for the U.S. Department of Energy by Battelle Memorial Institute 


\title{
DISCLAIMER
}

This report was prepared as an account of work sponsored by an agency of the United States Government. Neither the United States Government nor any agency thereof, nor Battelle Memorial Institute, nor any of their employees, makes any warranty, expressed or implied, or assumes any legal liability or responsibility for the accuracy, completeness, or usefulness of any information, apparatus, product, or process disclosed, or represents that its use would not infringe privately owned rights. Reference herein to any specific commercial product, process, or service by trade name, trademark, manufacturer, or otherwise, does not necessarily constitute or imply its endorsement, recommendation, or favoring by the United States Government of any agency thereof, or Battelle Memorial Institute. The views and opinions of authors expressed herein do not necessarily state or reflect those of the United States Government or any agency thereof.

\author{
PACIFIC NORTHWEST LABORATORY \\ operated by \\ BATTELLE MEMORIAL INSTITUTE \\ for the \\ UNITED STATES DEPARTMENT OF EVERGY \\ under Contract DE-AC06-76RLO 1830
}

Printed in the United States of America

Available to DOE and DOE contractors from the

Office of Scientific and Technical Information, P.O. Box 62, Oak Ridge, TN 37831; prices available from (615) 576-8401. FTS 62€-8401.

Available to the public from the National Technical Infismmation Service, U.S. Department of Commerce, 5285 Port Royal Rd., Springfield, VA 22161.

NTIS Price Codes, Microfiche A01

Printed Copy

\begin{tabular}{cr}
\hline Price Code & Page Range \\
\hline A02 & $1-10$ \\
A03 & $17-50$ \\
A04 & $57-75$ \\
A05 & $76-100$ \\
A06 & $107-125$ \\
A07 & $126-150$ \\
A08 & $151-175$ \\
A09 & $176-200$ \\
A10 & $201-225$ \\
A11 & $226-250$ \\
A12 & $251-275$ \\
A13 & $276-300$ \\
A14 & $301-325$
\end{tabular}

\begin{tabular}{cc}
\hline Price Cocle & Page Range \\
\hline A15 & $326-350$ \\
A16 & $351-375$ \\
A17 & $376-400$ \\
A18 & $401-425$ \\
A19 & $426-450$ \\
A20 & $451-475$ \\
A21 & $476-500$ \\
A22 & $507-525$ \\
A23 & $526-550$ \\
A24 & $551-575$ \\
A25 & $576-600$ \\
A99 & $601-U p$
\end{tabular}


PNL -7301

UC -405

LOOKING AT LARGE DATA SETS USING BINNED DATA PLOTS

D. B. Carr

April 1990

Prepared for the U.S. Department of Energy under Contract DE-ACO6-76RLO 1830

Pacific Northwest Laboratory Richland, Washington 99352 


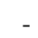

$-$.

-

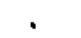

, 


\section{SUMMARY}

This report addresses the monumental challenge of developing exploratory analysis methods for large data sets. The goals of the report are to increase awareness of large data sets problems and to contribute simple graphical methods that address some of the problems. The graphical methods focus on two- and three-dimensional data and common tasks such as finding outliers and tail structure, assessing central structure and comparing central structures. The methods handle large sample size problems through binning, incorporate information from statistical models and adapt image processing algorithms. Examples demonstrate the application of methods to a variety of publicly available large data sets. The most novel application addresses the "too many plots to examine" problem by using cognostics, computer guiding diagnostics, to prioritize plots. The particular application prioritizes views of computational fluid dynamics solution sets on the fly. That is, as each time step of a solution set is generated on a parallel processor, the cognostics algorithms assess virtual plots based on the previous time step. Work in such areas is in its infancy and the examples suggest numerous challenges that remain. 


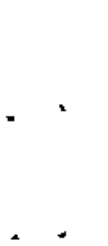




\section{CONTENTS}

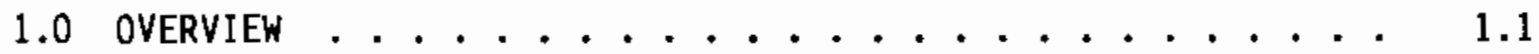

2.0 EXPLORATORY ANALYSIS OF LARGE DATA SETS . . . . . . 2.1

3.0 THE BINNING APPROACH TO LARGE DATA SETS PROBLEMS . . . . 3.1

4.0 GEOMETRIC, ALGORITHMIC AND VISUAL PROPERTIES

OF HEXAGONS AND TRUNCATED OCTAHEDRONS ........... 4.1

4.1 MERITS OF HEXAGONS AND TRUNCATED OCTAHEDRONS . . . . 4.1

4.2 MERITS OF SQUARES AND CUBES . . . . . . . . . 4.2

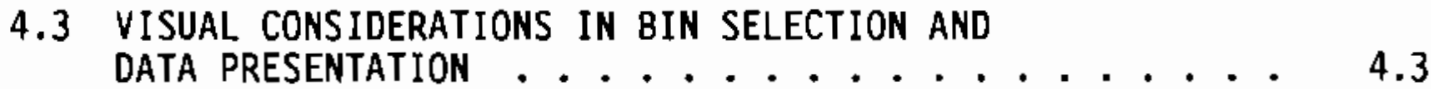

5.0 EXAMPLES INVOLVING HEXAGON BINS ................ 5.1

5.1 OUTLIERS AND DENSITY PATTERNS . . . . . . . . 5.1

5.2 FUNCTIONAL RELATIONSHIPS WITH
ONE INDEPENDENT VARIABLE $\ldots \ldots \ldots \ldots . \ldots . \ldots . \ldots$

5.3 FUNCTIONAL RELATIONSHIPS WITH
TWO INDEPENDENT VARIABLES .............. 5.7

5.4 CONDITIONED BIVARIATE DENSITY PLOTS . . . . . . 5.11

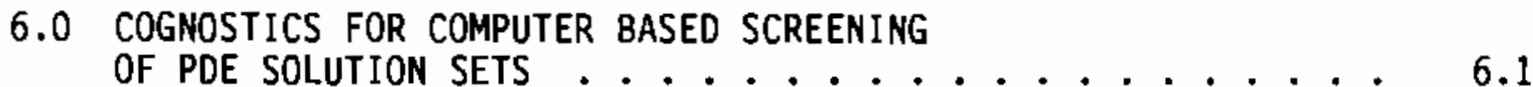

6.1 BACKGROUND ON PDE SOLUTIONS SETS . . . . . . . 6.2

6.2 DenSitY BASEd ARM DETECTION . . . . . . . . 6.6

6.3 PRACTICAL CONSIDERATIONS ................. 6.11

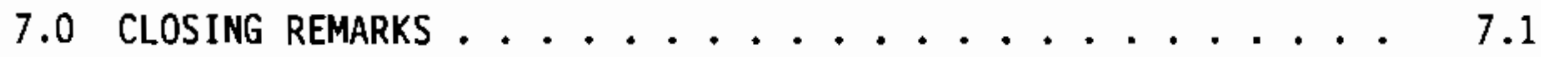

8.0 ACKNOWLEOGMENTS . . . . . . . . . . . . . . 8.1

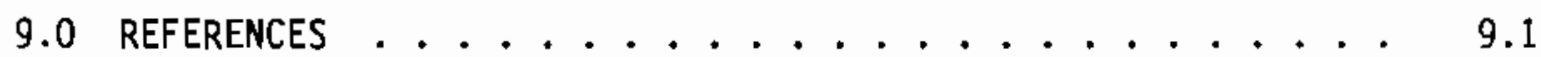




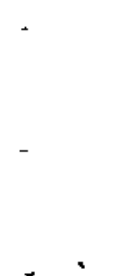

,

.

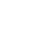

. 


\section{FIGURES}

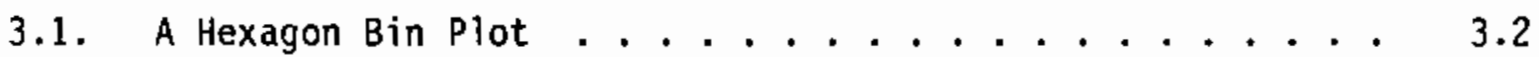

4.1. A Truncated Octahedron .............. 4.2

5.1. A Hexagon Bin Scatterplot Matrix .......... 5.2

5.2. A Hexagon Bin Scatterplot Matrix with Smoothes ..... 5.4

5.3. A Bivariate Binned Plot With Marginal Densities ...... 5.5

5.4. A Stereo-Pairs Added Variables-Plot . . . . . . . . 5.9

5.5. A Spatial Bivariate Plot ............ 5.11

5.6a. A Density Caricatures and Comparison Plot Matrix . . . . 5.14

5.6b. A Plot Showing Scales and Axis Labels.......... 5.15

6.1. A Scatterplot Matrix ............... 6.4

6.2. A Velocity vector Plot .............. 6.5

6.3. A Hexagon Bin Plot ............... 6.7

6.4. A Glass Melter . . . . . . . . . . . . . 6.8

6.5. A Skeleton Plot ................ 6.9

6.6. An Extreme Skeleton Plot ............ 6.10

6.7. A Stereo Pairs 3-D Skeleton Plot ........... 6.11 


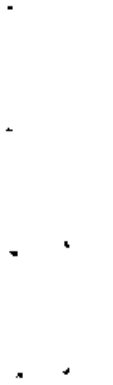

ฯ . 


\subsection{OVERVIEW}

This report presents practical graphical displays that can be used in the exploratory analys is of fairly large data sets. The starting point for most displays in this report is binned data. Working with binned data directly addresses large data set issues of computation and plotting speed. Almost everything that can be done with the original data can be done faster with binned data. Further, working with binned data allows image processing algorithms to be adapted and applied to bin cells. Thus tools can be brought to bare that are not traditionaliy associated with exploratory data analysis.

A previous paper by Carr, Littlefield, Nicholson and Littlefield (1987) discusses the exploration of large data sets using scatterplot matrices. This report, which is a sequel, provides new low dimensional displays and suggests new applications. In addition, this report is intended to encourage research in three areas. The first broad research area develops exploratory analysis tools for large data sets. It seems scarcely yesterday that EDA tools consisted of one outstanding mind, a four color pen, and a pad of legal size lined yellow paper. However the computing revolution is in full swing. Today's data rich environment as described by Hahn (1989) is soon to be followed by tomorrow's mind boggling data collection rates. The planned NASA earth observing station will handle 1 to 10 terabytes of data per day. If such data sets are to be examined in ways other than by use of narrowly focused, preconceived algorithms, then it is high time for statistical analysts to directly address the topic. Until exploratory tools are developed and applied, many details like the hole in the ozone Tayer are likely to remain undiscovered.

The second narrower research area develops better graphical methods for representing low dimensional data. Given some excellent books on graphics methods, such as the extensive catalogue of Bertin (1983), one might think that little remains to be done. However, rapid scanning of images made possible by modern workstations and video recordings tests our visual interpretive system. Plots need to be simplified, standards and 
language developed, and analysts trained before we have a fighting chance of passing the test. Bertin raises the question "what level of simplification is best?" For exploratory analysis this appears to be an open question in two dimensions and above.

The third research area develops cognostics, computer guiding diagnostics. In reality most collected data will not be subject to exploratory examination. Consequently, developing tools that extend the reach of trained analysts is important. Cognostics algorithms can be used to prioritize the plots to be examined by analysts. This is not to say that today's algorithms are able to replace the eye-brain system. Rather, in the face of overwhelming amounts of data, even primitive tools are helpful. The development and application of cognostics is in its infancy. While application of cognostics is dependent on computing speed, the greatest current need is for involvement of researchers and practitioners from the areas of data analysis, graphics design, cognitive psychology and image processing.

This report, a small attack on a large problem, is organized as follows. Section 2 describes the challenge of examining large data sets. Section 3 elaborates on the binning approach to large data set examination. Section 4 presents the particular binning emphasized in this report: hexagon binning in two dimensions and truncated octahedron binning in three dimensions. The majority of graphical examples are presented in Section 5 in the context of exploratory analysis tasks. Most of the applications are within the traditional scope of statistical analysis. Section 6 introduces cognostics in the novel setting of screening solution sets from computational fluid dynamics models. The volume of data is sufficient that cognostics are computed in parallel just behind each time step of the solution set and "interesting" plots are saved. The structure in such solution sets is so different that statistical analysts who have examined thousands of 2-D scatterplots will see new patterns. Section 7 wraps up with comments about the availability of algorithms and further challenges.

This report omits discussion of two important areas of exploratory graphical display. First, the report bypasses discussion of univariate 
exploratory analysis. The assumption is made that univariate exploratory analysis is familiar. It is presumed that exploratory analysis starts with univariate displays to learn about the variables, their ranges, their categories, and their distributions. Second, the report says little about the transformation of variables. This important issue is also a topic in its own right. 


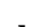




\subsection{EXPLORATORY ANALYSIS OF LARGE DATA SETS}

The exploratory analysis of large data sets is a relatively new area. Until the age of computing, it was difficult to collect large data sets, difficult to analyze the data sets and almost impossible to make such data sets widely available to interested parties. In the last twenty years the situation has changed dramatically. Monitoring studies now routinely produce large data bases. Examples of such studies include monitoring the earth's functioning, its natural resources and the continued impact of mankind on its environment. Additional monitoring studies include studies of human populations in terms of behavioral characteristics such as product consumption and disease transmission. A second class of studies that potentially generates large data sets are computer simutations. The word potential is important because frequently the intermediate results and portions of "final" results are not stored.

The computing revolution has altered the analysis of large data sets. In some areas such as image processing, image reconstruction, and large scale modeling, current capabilities would have been unthinkable twenty years ago. Nonetheless analysis capabilities seem to fall even further behind data collection capabilities. Further, the techniques analysts commonly use often lag behind new, computationally intensive analysis capabilities. This has been noted in the area of robust analysis but is equally true for exploratory analysis. The message of hope in this report is that exploratory methods can be developed if not for today's large data sets, at least for yesterday's. Sample sizes of a few thousand cases should be grist for the classroom mi11. Students and professional data analysts should be trying their hand at exploring such data sets and developing new methods. The classical techniques of experimental design, sampling, and inference can be mixed with powerful computer modeling and visualization techniques to produce new insights.

The computing revolution has made many of yesterday's large data sets available. Many of the examples in this report are based on data sets obtained from government agencies. Almost by definition of their existence, 
these data sets are important. Institutions that devote so much effort to data collection and production of preplanned tables and analyses often have few resources left for data exploration. Their data sets are begging for creative, exploratory anatysis. 


\subsection{THE BINNING APPROACH TO LARGE DATA SETS PROBLEMS}

In broad terms the problems with large data sets relate to large sample sizes, many variables (dimensionality) and the complexity of relationships among the variables. Presuming that data access problems have been overcome, large sample sizes translate into problems of computing speed, plotting speed, and overplotting. The approach in this report is to use binning to address these problems. Almost everything that is done with raw data can be done faster with binned data and case weights.

Binning directly attacks the problem of overplotting. Figure 3.1 shows a hexagon binned plot. The cases have been binned in hexagon regions and hexagon symbols are plotted in these regions. The area of each hexagon symbol is an increasing function of the population size. Sometimes a log scale or other symbol scaling transformation is required to accommodate the range of the counts. For simple situations, each hexagon symbol is shifted from the bin center toward the center of mass for the data within the bin with the constraint that the symbol not plot outside the binning region. This reduces the visual dominance of the binning lattice and the plot looks very much like an ordinary scatterplot. Such plots can gracefully accommodate thousands of points and are easily printed using laser printers.

Binning has two basic limitations. First, binning sacrifices resolution. Sometimes plots of the raw data will reveal interesting fine structure that is hidden by binning. Examples in this report are oriented toward quick overviews so the binning is coarse. However, advantages from binning often outweigh the disadvantage from lost resolution. Large data sets provide the opportunity to obtain quality density estimates using high resolution binning ( $5 \operatorname{cott}$ and Terrel 1986) and modern visualization techniques allow rapid exploration of precomputed bivariate density estimates (Carr, Littlefield, Nicholson, and Littlefield 1987).

Second, binning does not extend well to high dimensions. With reasonable univariate resolution, say 50 regions each covering $2 \%$ of the range of the variable, the number of cells for a mere 10 variables is 


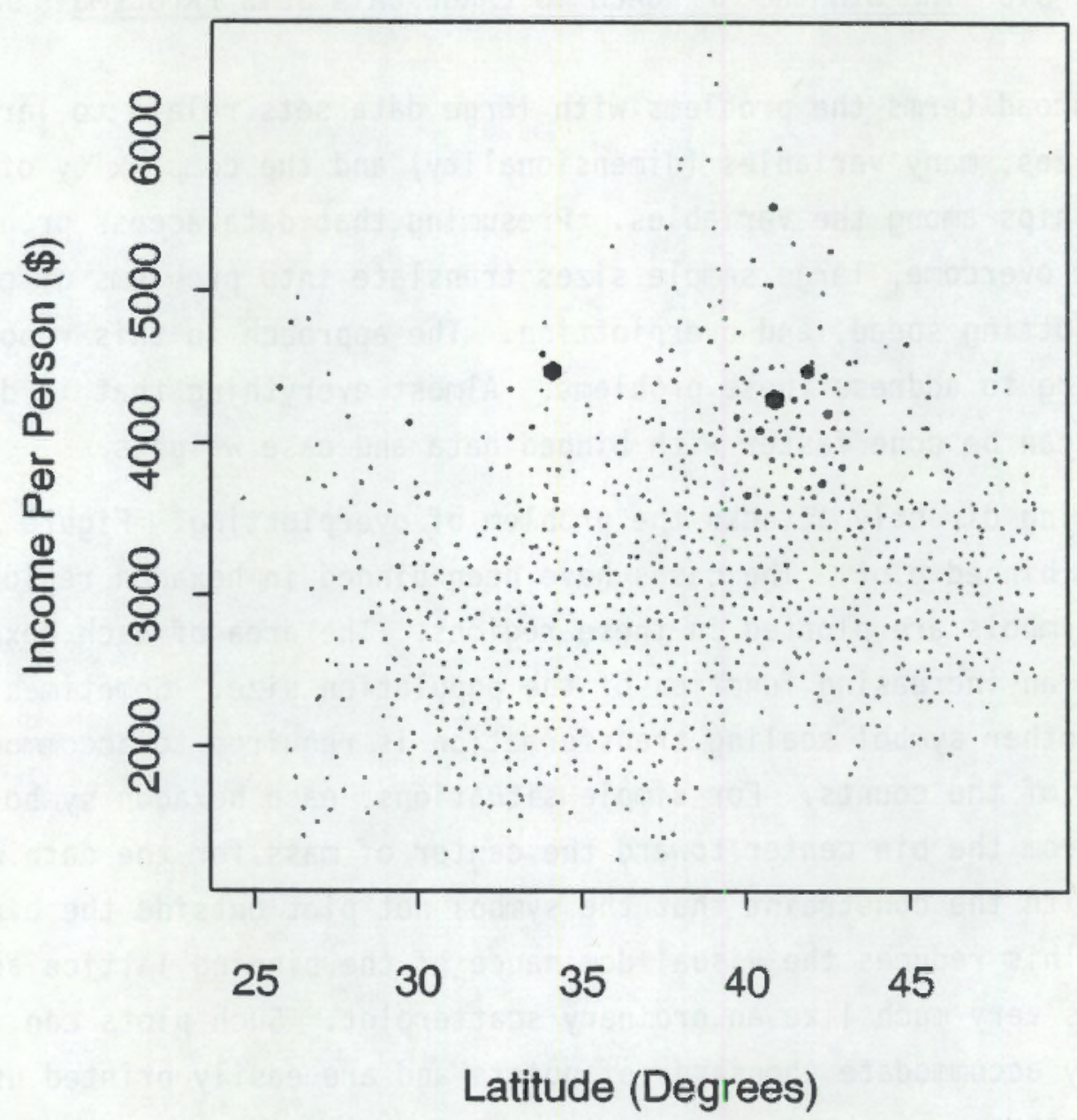

FIGURE 3.1. A Hexagon Bin Plot. The county based data set comes from the Bureau of Census. Interpretation is similar to an ordinary scatterplot. For example note that low income is associated with southern (low latitude) counties. As for construction, the hexagon symbols are plotted inside each hexagon binning region. The area of each symbol is a linear function of the square root of the county population in the binning region. A minimuin symbol size represents all small population counts. To the extent possible, the symbols are moved toward the data center of mass in each binning region. This reduces distraction induced by the binning lattice. 
exceedingly large. For uniformly distributed data, it would take a huge sample size to fill a respectable fraction of the cells. The message is not so much that binning is bad but that high dimensional space is big. The complement to the curse of dimensionality is the blessing of large samples. Even in two and three dimensions having lots of data can be very helpful when the observations are noisy and the structure non-trivial.

While large samples sizes are conceptually ideal for identifying high dimensional relationships, this report focuses on finding the occasional low dimensional display that leads to understanding. Thus the existence of many variables and the complexity of relationships among variables translates into a staggering quantity of low dimension plots. Included are not only plots of variable pairs or triples, but also plots conditioned on the values of other variables. The problem of too many plots cannot be solved. However, rapid visual assessment and cognostics, as discussed in Sections 5 and 6 , at least attack the problem. 


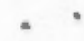

-
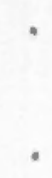


\subsection{GEOMETRIC, ALGORITHMIC AND VISUAL PROPERTIES OF HEXAGONS AND TRUNCATED OCTAHEDRONS}

Since binning is a fundamental concept in this report, some background may be helpful. Binning is not a new idea. Square and cube bins are occasionally used in data analysis. Thus the emphasis here on hexagons and truncated octahedrons warrants some explanation.

Section 4.1 describes merits of hexagon and truncated octahedron bins and Section 4.2 presents merits of square and cube bins. These two sections provide background concerning bin geometry and the algorithms that deal with bins. The decision to use hexagon and truncated octahedron bins is primarily based on visual considerations as described in Section 4.3.

A clear image of the bin geometry helps in following the discussions. A perspective view of the truncated octahedron is shown in Figure 4.1. The vertices can be generated by considering all permutations of the point coordinate $(0,+-1,+-2)$. Thus the truncated octahedron has 24 vertices and 14 sides; eight are hexagons and six are squares.

\subsection{MERITS OF HEXAGONS AND TRUNCATED OCTAHEDRONS}

Hexagons and truncated octahedrons are polytopes with three important properties. First, the polytopes are tessellations. Hexagons partition the plane and truncated octahedrons partition space. Second, these polytopes have the smallest dimensionless second moment of all dimension filling polytopes (Conway and Sloane 1982a). In other words, the polytopes are the closest to being circular and spherical, respectively. Scott's (1985) result, that density estimates using hexagons have somewhat smaller mean square error than those using squares of equal area, is a consequence of this property.

Third and perhaps most important, hexagons and truncated octahedrons have a well defined number of neighbors. A hexagon shares a face and a vertex with six neighbors. In contrast, a square has four neighbors when 


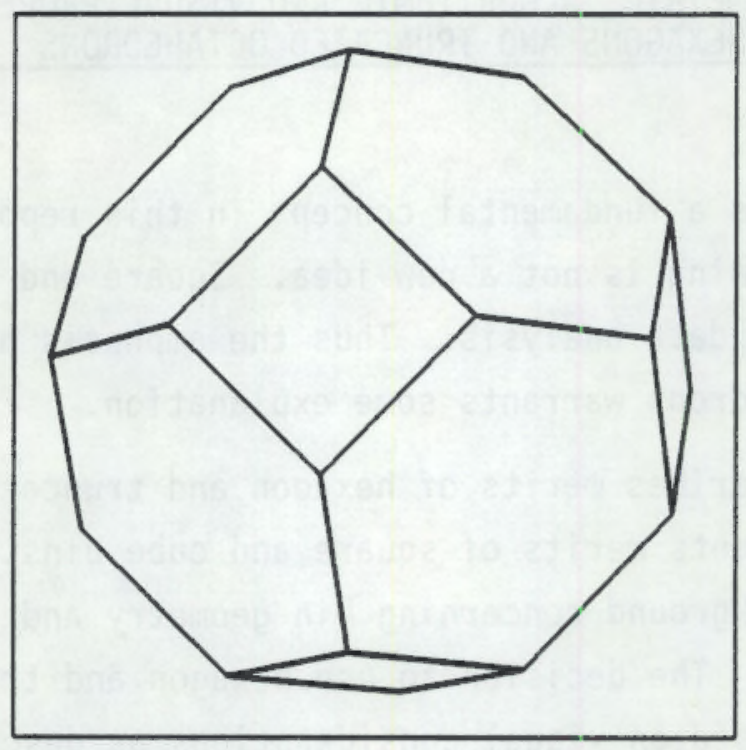

FIGURE 4.1. A Truncated Octahedron. Only the front surfaces are shown. Truncated octahedrons are the "roundest" polytopes that form a regular tessellation of three space.

considering shared faces and eight neighbors when considering shared vertices. Similarly the truncated octahedron has 14 neighbors while the cube has 6 or 18 or 26 neighbors depending on whether shared faces, edges, or vertices are considered. The number of neighbors may not be crucial in terms of direct visualization, but the geometry clearly impacts computation. For example, when assessing conditions based on neighbor occupancy, $2^{\star \star} 14=16,384$ possibilities is a manageable number for a lookup table but $2 \star \star 26$ is not.

\subsection{MERITS OF SQUARES AND CUBES}

Squares and cubes have four advantages over hexagons and truncated octahedrons. First, binning methods for squares and cubes are faster. Closer examination shows that the binning methods are related and the speed difference is well defined. Conway and Sloane (1982b) indicate that hexagons are the Voronoi (near neighbor) regions for the union of two rectangular lattices. The two lattice spacings are identical but one lattice is shifted to split the cells of the other. Similarly, truncated 
octahedrons are the near neighbor regions of a body centered cube lattice. This lattice can be viewed as the union of two cube lattices, one of which is shifted. Good binning algorithms then find one candidate lattice point for each of two rectangular (cube) lattices and end with one or two distance calculations to determine which of the two candidate lattice points is closer. Thus, ignoring reading and scaling overhead, square and cube binning is always faster by a factor of two plus at least one distance calculation and a comparison.

The second advantage of squares and cubes relates to processing data structures. Squares and cubes can be treated conceptually as one array. The hexagon and truncated octahedron structures must be handled conceptually as two arrays corresponding to the two shifted lattices. Whatever the storage implementation (for example see Fabbri 1984 and Dekinder, Blackman and Gibson 1982), hexagons and truncated octahedrons require either extra bookkeeping to keep track of the shift between the two lattices or special algorithms.

The third advantage of squares and cubes relates to graphics devices. Standard raster devices have rectangular pixels. Thus straight lines look jagged except along two orthogonal directions. Similarly most plotters have preferred orthogonal directions. Except for very high resolution devices, plots involving properly oriented rectangles look cleaner in terms of the lines.

The fourth advantage of square and cube bins involves nesting. Small squares can cleanly partition large squares, but small hexagons leave ragged edges when partitioning a larger hexagon. A similar result holds for cubes versus truncated octahedrons.

\subsection{VISUAL CONSIDERATIONS IN BIN SELECTION AND DATA PRESENTATION}

Many algorithmic considerations favor square and cube bins over hexagon and truncated octahedron bins. However today's computing power allows the choice to be made based on human visual considerations. Humans, with their sense of balance, have a strong reaction to horizontal and vertical lines. 
The half-toning process avoids horizontal and vertical lines to facilitate interpretation of coarse dots as gray level. Similarly Carr, Littlefield, Nicholson and Littlefield (1987) conjecture that the hexagons with three non-orthogonal lines through opposite neighbors help the data speak by reducing emphasis on preferred visual lines. Visual lines that are artifacts of plot construction constitute a distraction and should be avoided. Horizontal and vertical lines are particularly distracting. Visual lines are created when bin symbols are plotted in the binning regions. That is, visual lines are generated by the location of the symbols. While overplotting should be controlled, plotting symbols near their center of mass rather that at bin centers helps break up the visual lines.

In addition to plotting location, many symbols suggest visual lines by their shape. For example, identically oriented squares suggest two orthogonal directions. Hexagon and truncated octahedron bins complement round symbols. Of course, round symbols can be used for square and cube bins but that wastes plotting space. The wasted space creates a distracting pattern. Hexagons and truncated octahedron bins are chosen here as preferred binning regions both because the directions parallel to the opposite faces, 3 and 7 respectively, are not orthogonal and because the bins complement round symbols 


\subsection{EXAMPLES INVOLVING HEXAGON BINS}

This section provides examples of plots that involve the use of hexagon binning. The simplest cases involve looking for density patterns (Section 5.1) and functional relationships (Section 5.2) in bivariate plots. Section 5.3 addresses the situation with two independent variables and one or more dependent variables. Section 5.4 returns to the examination of density patterns in the context of comparing multiple bivariate plots.

\subsection{OUTLIERS AND DENSITY PATTERNS}

The identification of outliers often provides a beginning to important detective work. With large data sets, many tasks can be approached by sampling. However, outliers are typically few in number so have very low probabilities of being observed under simple sampling schemes. Consequently, plots showing all the data are particularly important for outlier identification. Figure 5.1 shows a binned scatterplot matrix based on pixel intensities from the seven spectral bands of a $512 \times 512$ section of a Landsat image. Each plot represents over a quarter of a million intensities. To accommodate the large range of counts, the hexagon symbol size is a linear function of $\log$ base ten of the counts with a minimal symbol for counts of one. Outlying cells are evident in the bivariate plots. In fact, a striking feature is the lack of outlying cells in the Band 2 versus Band 3 plot. Note that few of the outliers would be evident in univariate histograms. By extension, more outliers would likely be found by examining higher dimensional plots. The outliers and non-gaussian distributional shapes suggest the use of robust variants of principal components and other image enhancement algorithms.

\subsection{FUNCTIONAL RELATIONSHIPS WITH ONE INDEPENOENT VARIABLE}

A second common task in exploratory data analysis is the identification of functional relationships. The functional relationship between band 1 and band 2 in Figure 5.1 is quite evident. When observations vary little 


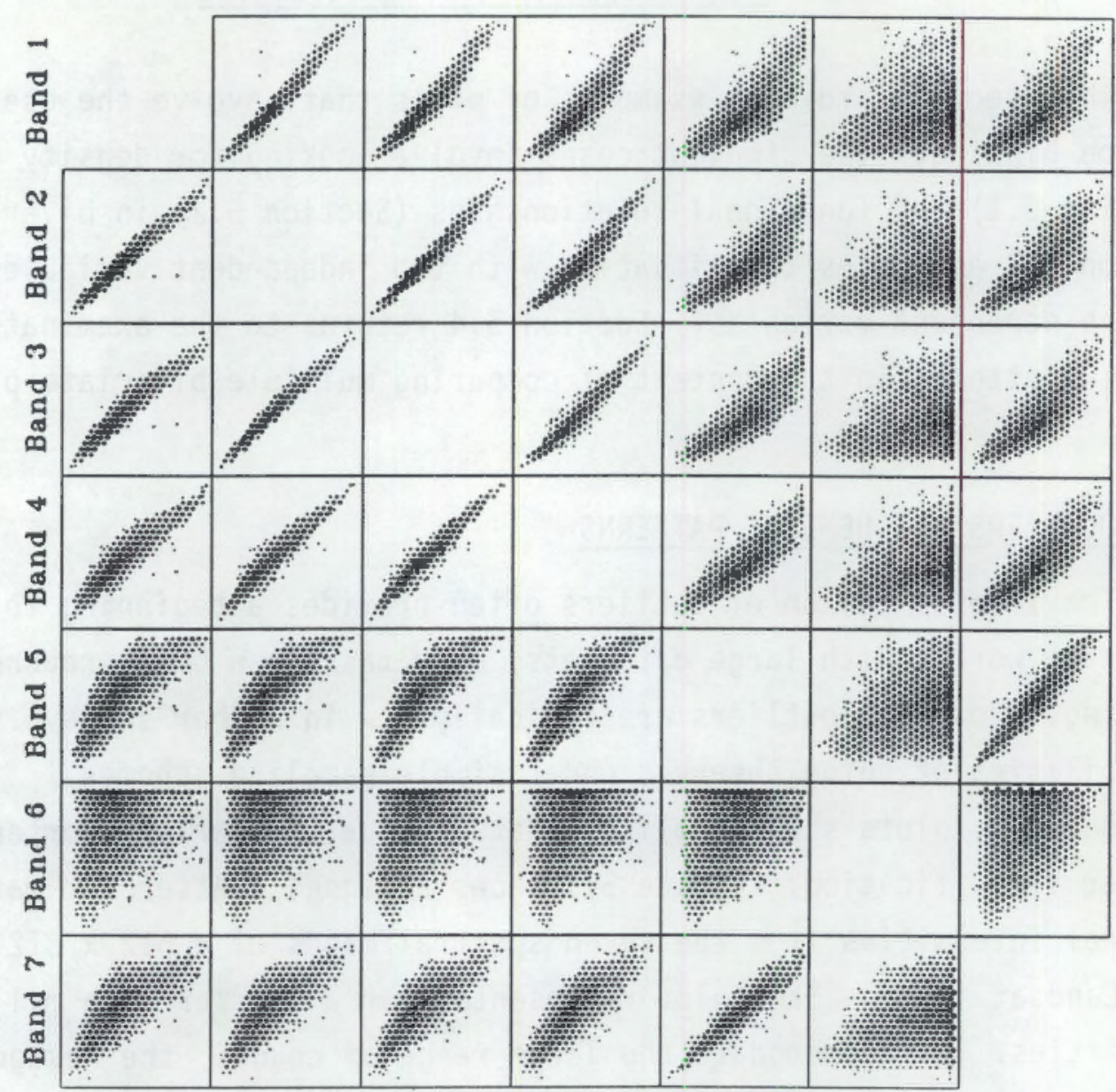

Band 1 Band 2 Band 3 Band 4 Band 5 Band 6 Band 7

FIGURE 5.1. A Hexagon Bin Scatterplot Matrix. The data set is a $512 \times 512$ Landsat image of a near ground zero at the Nevada Test Site. The hexagon symbol area is linearly related to the $\log$ base ten of the number of pixels in each binning region. Each plot represents more the 250,000 pixels. The spectral bands correspond to different wave lengths of light. For example Band 1 covers 0.45 to $0.52 \mu \mathrm{m}$ and Band 7 covers 2.08 to $2.35 \mu \mathrm{m}$. The bands are in wave length order except for Band 6, which covers 10.4 to $12.5 \mu \mathrm{m}$. The plotting axis for each band is based on the observed range of pixel intensities. Interesting plot features include the cutoff of pixel intensity values for Band 6 (the thermal band) and the bivariate outliers that would not be evident as outliers in univariate plots. 
about the functional form relative to the variation in the functional form, the eye can easily fit a curve. When the functional form explains only a small part of the variation, Cleveland and McGill (1984b) recommend plotting a smooth. Smoothes approximate the functional relationship by providing local estimates of $E(y \mid x)$ over the range of $x$. Computing smoothes for binned data is analogous to computing smoothes for the original data except the smoothing needs to accommodate bin count weights.

Figure 5.2 shows smoothes for noisy data. Before describing the plot some background is helpful. The data set consists of melanoma rates for contiguous U.S. counties (excludes Alaska and Hawaii) obtained from Riggan et al. (1983), some 200 demographics variables (in including elevation) obtained from the Bureau of the Census, and insolation data obtained from the Solar Research Institute. Melanoma is a lethal form of skin cancer. The death rate per million for white males in the U.S. has increased from 13 per million in 1950-1959 to 24 per million in 1970-1979. The increase is a cause for concern particularly in view of current discussion about reduction in the ozone layer. The ozone layer absorbs ultraviolet light and ultraviolet light exposure is generally accepted as a major factor in the occurrence of melanoma.

This particular epidemiology study assumed that melanoma rates would be related to latitude. The further from the equator, the less the ultraviolet exposure. The study began by suspecting elevation as a factor influencing melanoma rates. The conjecture was that at high elevations less atmosphere is present to absorb ultraviolet light so the melanoma rates would be higher.

A striking feature of the data set is the range for county population. The binned plot in Figure 5.3 conveys some of this information and the marginal densities show the domination of cites at two latitudes. Thus county population should be considered as a factor in estimate variability and may be confounded with important independent variables. There is an interplay between statistical models and graphics, so it is advantageous to think about models before making a series of plots. Here the goal is to model age adjusted death rates $r(i)$ where $i$ indexes the counties. An 


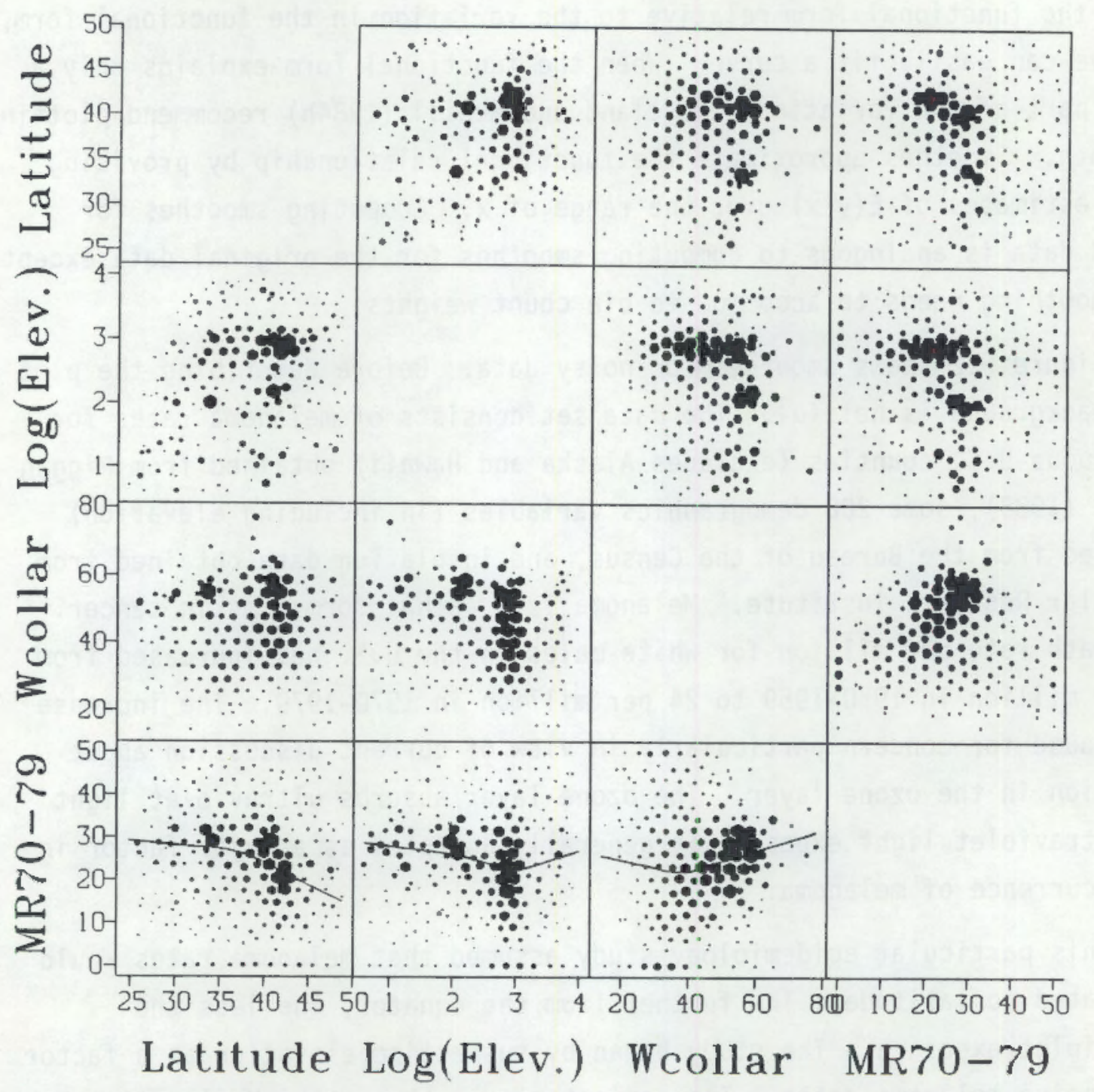

FIGURE 5.2. A Hexagon Bin Scatterplot Matrix with Smoothes. The dependent variable is county-based age-adjusted male melanoma mortality rate per million based on the 1970-1979 decade. Values above 50 are winsorized at 50 to provide resolution in the rest of the plot. The county-based independent variables are latitude, $\log$ base 10 of elevation and percent white collar workers. County population for the 3056 U.S. counties is the variable binned and the sum represented by hexagon size. The large variation in the plots obscures the relationship of melanoma mortality rate to latitude and percent white collar. The relationship that varies by more than ten per million is brought out by plotting population weighted smoothes. 


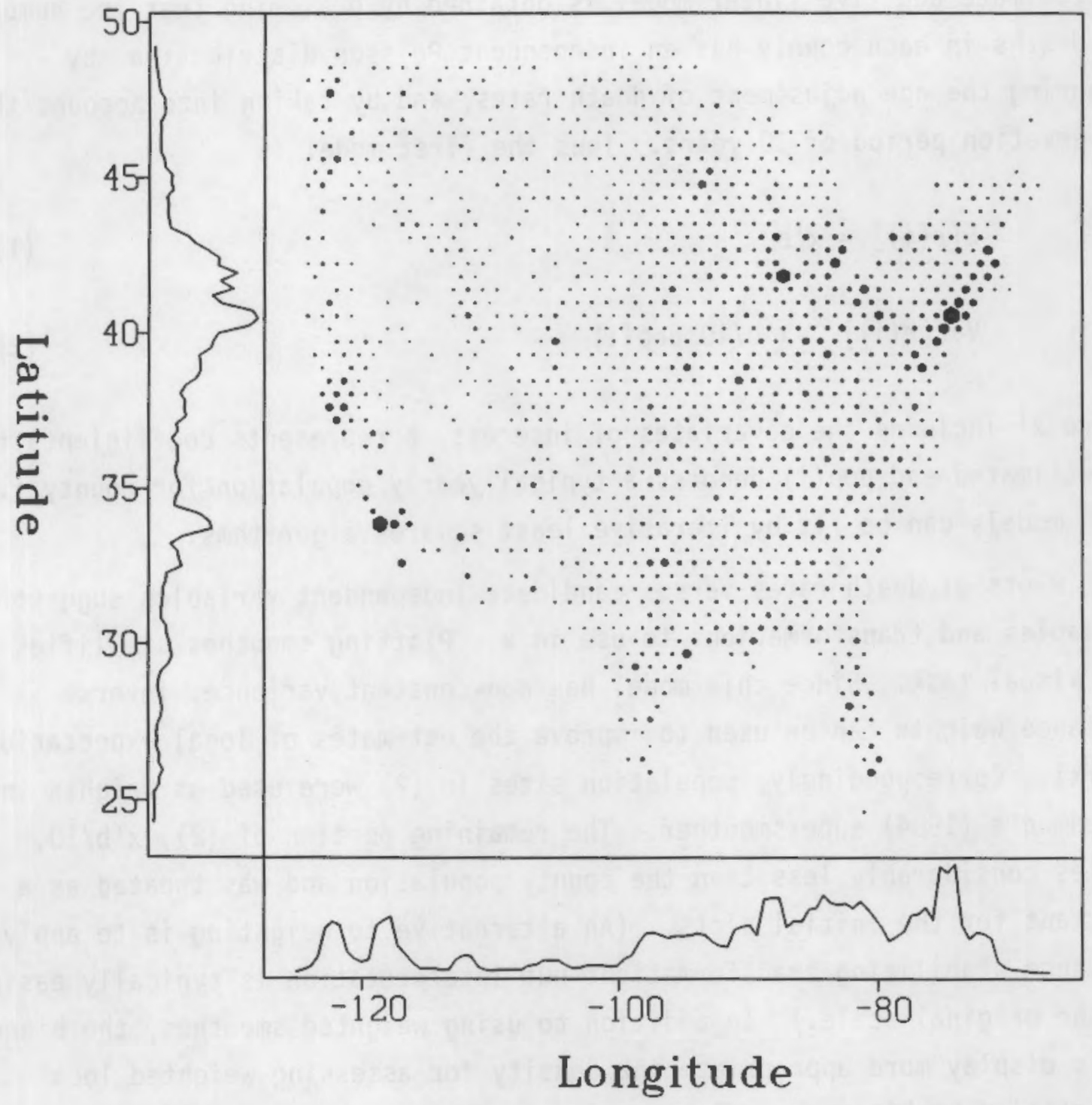

FIGURE 5.3. A Bivariate Binned Plot With Marginal Densities. The quantity binned is county population. The marginal density plot for latitude has two dominant modes. Large cities contribute substantially to these modes. Mortality variations apparently due to latitude may be confounded with other differences between a few large cities. 
approximate additive linear model is obtained by presuming that the number of deaths in each county has an independent Poisson distribution, by ignoring the age adjustment of death rates, and by taking into account the observation period of 10 years. Thus the first model is

$$
\begin{aligned}
& E[r(i)]=x^{\prime} b \\
& \operatorname{Var}[r(i)] \sim x^{\prime} b / 10^{*} p o p(i)
\end{aligned}
$$

where $x^{\prime}$ includes the covariates of interest, b represents coefficients to be estimated and pop(i) denotes a typical yearly population for county $i$. Such models can be fit by iterative least squares algorithms.

Plots of death rates versus candidate independent variables suggest variables and transformations to use in $x$. Plotting smoothes simplifies the visual task. Since this model has non-constant variance, inverse variance weights can be used to improve the estimates of local expectation, $E(y \mid x)$. Correspondingly, population sizes in (2) were used as weights in Friedman's (1984) supersmoother. The remaining portion of (2), $x^{\prime} b / 10$, varies considerably less than the county population and was treated as a constant for the initial plots. (An alternative to weighting is to apply variance stabilizing transformations but interpretation is typically easier in the original scale.) In addition to using weighted smoothes, the binned plots display more appropriate ink density for assessing weighted local expectation by binning population sizes rather than by the number of counties as shown in Figure 5.2 .

The existence of a relationship in the plot of mortality rate versus latitude was not a surprise but the plot suggests a quadratic relationship rather than a linear one. The plot of rate versus percent white collar was a surprise. The variation in the percent white collar smooth was about the same as in the latitude smooth. Since there is a tendency for the percent white collar to increase with latitude, failure to account for such a variable could lead to an underestimate of the important latitude effect. 
The study began with a suspicion about a relationship between elevation and mortality rate. The plot of mortality rate versus elevation is shown on a $\log$ base ten scale with sea level values replaced by $\log 10(4)$. The pattern was a disappointment, suggesting a slight decrease moving from the sea level to the large populations at 1000 feet and then a slight increase at higher elevations. If elevation is related to mortality rates, it is likely well disguised by behavioral patterns (clothing and exposure) that differ between near sea level large cities and high mountain small towns.

The simple plots in 5.2 contain additional suggestions. Note that the zero rates are separated from the body of the data. The separation suggests a possible reporting problem associated with small counties. A statistical test shows the number of zeros is plausible but that doesn't totally answer the question raised by the lack of continuity. Note the tremendous variability in the plots. The prospect of explaining most of the variability does not look good, so other, more controlled studies seem advisable. Finally, note the reduction in variability for high values of percent white collar. The plot warns of homogeneity problems in more detailed modeling efforts.

\subsection{FUNCTIONAL RELATIONSHIPS WITH TWO INDEPENDENT VARIABLES}

A recurrent task in exploratory analysis is assessing the relationship between a dependent variable and two independent variables. The task involves the fitting and visualization of surfaces. Several multivariate smoothers are available to assist in the task. Two strong candidates are thin plate smoothing splines (Wahba 1986) and local regression smoothes (Cleveland and Devlin 1988). For large data sets, some adaptation must be made. The number of operations is $O\left(n^{\star \star 3}\right)$ for the smoothing splines algorithm and $O(n)$ for each data point for the local smoothing algorithm. The methods could be modified to handle binned data and produce central tendency and variability estimates at bin centers of occupied bins.

If sophisticated algorithms are not available, it is possible to get started looking at the data by accumulating central tendency and variability 
statistics on the dependent variable $z$ for hexagon cells based on $x$ and $y$. If the binning process itself does not provide enough smoothing, local inverse variance weighted averaging of neighboring cells will usually suffice. Then the visual examination process can proceed.

Numerous methods are available for displaying surfaces, including contour plots and perspective plots. A1l methods have their deficiencies. Contour plots do not have direct visual impact unless enhanced by line thickness or pseudo-color. Even then, the dependent variable is broken into a few categories and represented by visual variables (especially color) that have low accuracy of extraction (Cleveland and McGill 1984a). Perspective plots provide better overviews. However if a surface is rough, part of the surface is hidden. Further, the accuracy for assessing surface values is low because of the changing reference plane. A common problem for both types of plots is that they do not indicate the variability of local surface estimates except as can be inferred from their local instability.

The stereo pairs binned plot in Figure 5.4 addresses these problems. Figure 5.4 is a regression diagnostics plot for two added variables. The variables are from the melanoma data base as described in Section 5.1. In Figure 5.4, stereo depth shows adjusted mortality rate residuals. Stereo depth, while not assessed with great accuracy, provides a continuous and directly interpretable representation. The $x$ and $y$ coordinates in the plot correspond to adjusted independent variables, latitude squared and percent white collar workers, respectively. To convey the variability of the local surface estimate, the hexagon symbol size is chosen so that it relates inversely to the standard error of the local central tendency estimate. A small fixed hexagon size is used when only one observation is available in a cell and when the standard error is very large. Better methods may be devised for calling attention to tightly moored estimates and fuzzing the other estimates, but representing local variability is a step forward. 

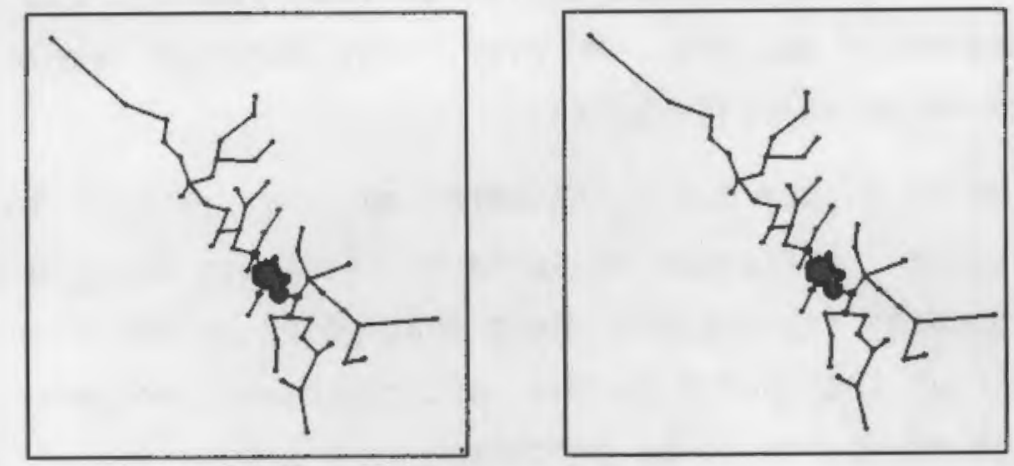

FIGURE 5.4. A Stereo-Pairs Added Variables-Plot. A stereoptican helps in fusing the images. However, some analysts can fuse the images by putting their nose between the images, by staring right between the images and by moving back slowly to about fourteen inches away.

The dependent variable is male melanoma mortality rate. The independent variables are latitude, latitude $\star \star 2$ and percent white collar. Latitude is presumed in the model. The adjusted variables, latitude**2 and percent white collar, are shown on the horizontal and vertical axes, respectively. For each hexagon binning region the average of mortality rate residuals is shown as stereo depth. Large positive values are closed to the viewer. For each binning region the hexagon symbol size is related to the inverse of the standard error of the mean. This gives more ink to values that are tied down. Single count cells and those with large standard errors are represented by a fixed minimal sized dot. Hexagons have been connected with a minimal spanning tree to facilitate stereo visualization. The plot suggests departures of a fitting plane. Further, the tip of a plane through the data does not appear to be well established in the direction orthogonal to the long axis through the data. Notice how simply this plot shows the basic structure of more than three thousand residuals.

Another challenge in the display of surfaces is to represent more than one surface. Skipping over the color bivariate plots criticized by Wainer and Francolini (1980), the most common approach is juxtaposition of two surface plots. Comparison of surfaces in juxtaposed plots is a difficult visual task unless the plots are very similar. In the case of contour plots part of the comparison difficulty becomes evident when superimposed plots are considered. When two colors or translucency 
techniques are available, perspective surface displays can be superimposed with some degree of success. However, that does not remove the inherent difficulties of perspective plots.

The plot in Figure 5.5 illustrates another approach to showing more than one surface. Estimates of surface values for hexagon binning regions are represented by ray angles. Rays that point to the right represent one surface and rays that point to the left represent another. The connecting dot helps the ray pairs to be processed as a single visual unit. Thus the plot can be scanned for unusual correlations. Since angles are textons (see Julesz and Bergen 1983) local departures from consistent angular patterns are picked up in preattentive vision. Bivariate anomalies are easy to find when coded as discrepant angles.

For assessing individual surface values, the ray is a reasonable symbol since angles have good accuracy of extraction (Cleveland and McGill 1984a). The extraction can be made more accurate by adding fixed orientation tics to the center dot in each bivariate symbol. Comparison of left and right angles is easy. Interpretation, of course, depends on the scales. How the scales are chosen may vary from problem to problem. One possibility is to add global distributional references by making horizontal vectors correspond to a measure of surface central tendency such as the median (see Carr, Nicholson, Littlefield, and Hall 1986). In Figure 5.5 straight down corresponds to $(0,0)$ and straight up to $(37.5,75)$ because the 1 to 2 ratio is easy to remember.

Figure 5.5 generalizes in two ways. First, information about the standard errors of local surface estimates can be added using ray length, gray level, or line thickness. The general strategy is to make the better estimate more pronounced. Second, if color is available for distinguishing rays, an additional surface or two can be added before the plot becomes hopelessly messy. Thus ray plots can encode much information. The main weakness of ray based surface plots is failure to give direct gestalt impression of the surface. For that purpose perspective views are typically superior. 


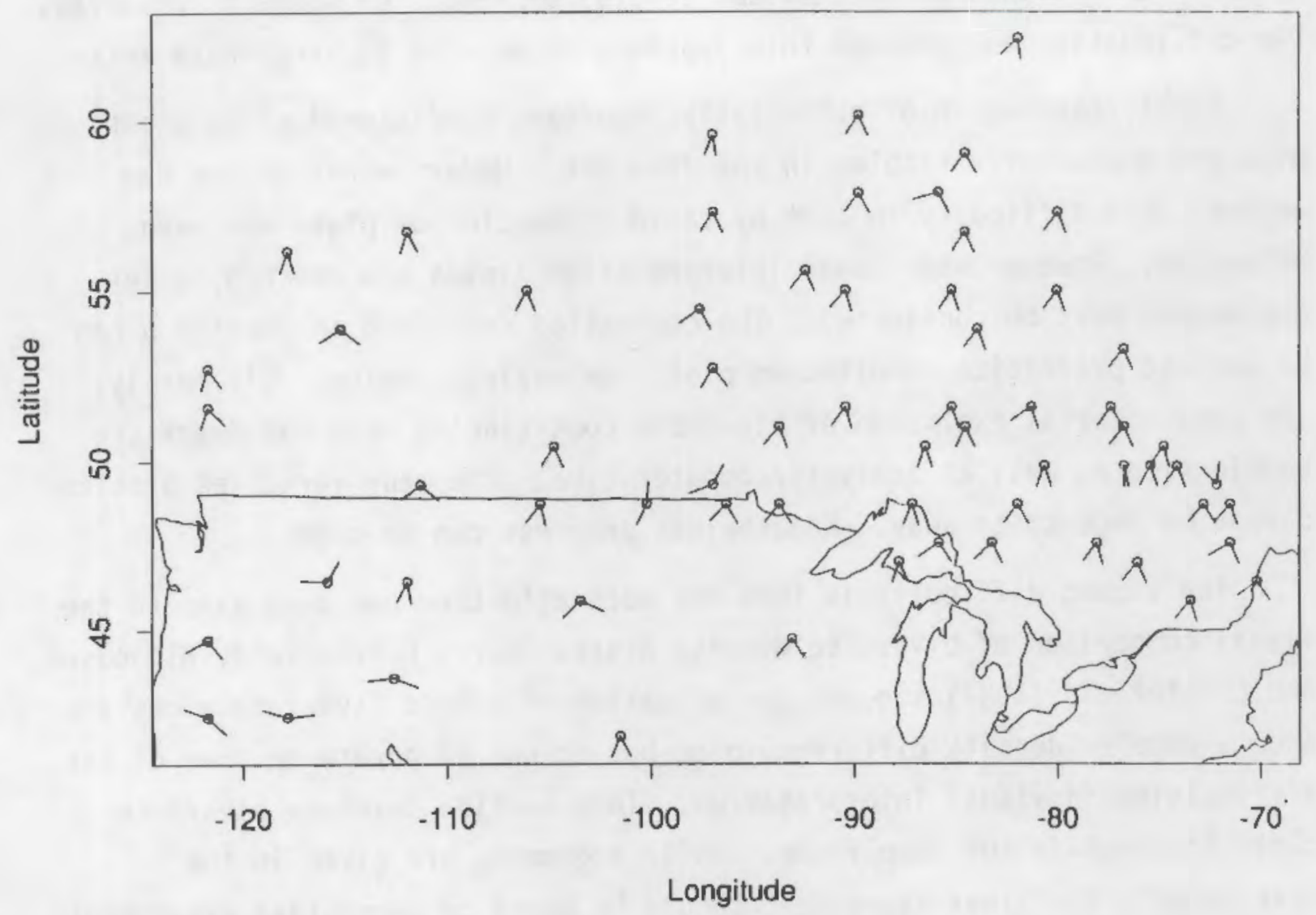

FIGURE 5.5. A Spatial Bivariate Plot. Rays pointing to the left and right represent Cesium-137 and Iodine-131 concentrations, respectively. Ray angles represent local average values based on 2000 observations taken after the Chernobyl reactor accident. Rays pointing straight down represent 0 parts per billion. Rays pointing straight up represent 37.5 and 75 parts per billion for Cesium and Iodine, respectively. General correlation patterns are evident and unusual bivariate correlations are quickly spotted. The plot raises several questions including one concerning the consistency of background corrections.

\subsection{CONDITIONED BIVARIATE DENSITY PLOTS}

When many variables are present, bivariate plots are often produced by ignoring the values of all other variables. Such bivariate plots often represent the composite of different bivariate distributions that are indexed by values of ignored variables. To explore the possibility of different distributions, a natural approach is to compare bivariate plots 
that are conditioned on categories, levels, or ranges of suspect variables. Two difficulties emerge when this approach is applied to large data sets.

First, the number of potentially important conditioned plots grows with the number of variables in the data set. Modern workstations can address this difficulty in part by rapid production of plots and even animation. However when human interpretation 1 imits are reached, other approaches must be considered. The cognostics described in Section 6 can be used to prioritize conditioned plots for analyst review. Ultimately, the combinatorial explosion of plausible conditioning sets can dwarf the sample size as well as analysts/computer time. The many variables problem cannot be made to go away. Nonetheless progress can be made.

The second difficulty is that not much attention has been give to the visual comparison of bivariate density plots. Carr, Littlefield, Nicholson, and Littlefield (1987) suggest the animation of binned bivariate plots and show a complex density difference plot but do not elaborate on some of the difficulties in visual interpretation. This section develops bivariate plots to simplify the comparison. While arguments are given in the development, the final assessment should be based on perceptual experiments so readers are encouraged to think carefully about the issues and alternative plots.

Before developing a visual representation for comparing bivariate distributions, consider the comparison of univariate distributions. For comparison of univariate distributions, the statistical graphics conmunity often juxtaposes boxplots. Note that the boxplot is a distributional caricature based on just five numbers (plus outliers). The boxplot brings out some features, such as the median and quartiles, while hiding other possible features of interest, such as bimodality. Juxtaposed boxplots allow easy visual comparison of the five numbers since corresponding numbers have similar representations from plot to plot, and since the visual representations (see Tukey's 1989 version) for each of the five numbers is easily distinguished. Further, the boxplot is a community standard (Frigge, Hoagl in, and Iglewicz 1989 discuss minor variations) so analysts have considerable experience in examining and interpreting such plots. The 
univariate situation suggests keeping things simple in the bivariate situation.

A logical starting place for a bivariate caricature is a high density contour that contains $50 \%$ of the observations. While this contour is used in what follows, a bivariate contour is not a simple entity in terms of visual assessment. By itself a contour can be more complex than a boxplot. The comparison of two contours is an additionally complex visual process because contours do not have corresponding parts. Papathomas and Julesz (1988), provide one approach to visual comparison by plotting two regions at different depths in stereo plots. The approach here is to provide an explicit visual representation of plot differences.

Seeing the proposed bivariate caricature plots helps fix ideas in the remaining discussion. Figure 5.6 a shows a matrix of plots involving groups that differ in two variables, age and sex. The shaded regions in the plots with odd row and column numbers are approximate $50 \%$ high density contours. The filled dot in each of these plots represents an estimate of the bivariate median. The plots with even row or even column number show differences between adjacent plots. The light shaded region represents the contour area left behind in the transition from the plot on the left (top) to the plot on the right (bottom). The dark shaded region represents the area added in the transition. The outlined cells with arrows show the movement of the center cells with the arrow pointing to the right (bottom) center cell.

Figure 5.6a uses data from the National Health and Nutrition Examination Survey I (NHANES I). In the survey, a probability sample of the U.S. population was given a medical exam, dietary questionnaire, and blood tests during the period 1971-1974. These 14,407 adults aged 25-79 have been followed for disease occurrence. The plot in Figure $5.6 \mathrm{a}$ is designed to provide information about variables, serum albumin (mg/dl) and transferrin saturation (\%), thought to be related to cancer occurrence. The ranges for the $50 \%$ contours are shown in Figure $5.6 \mathrm{~b}$. The pattern in Figure $5.6 \mathrm{a}$ is pretty clear. Males start with higher albumin and transferring saturation values than women. The male albumin values 


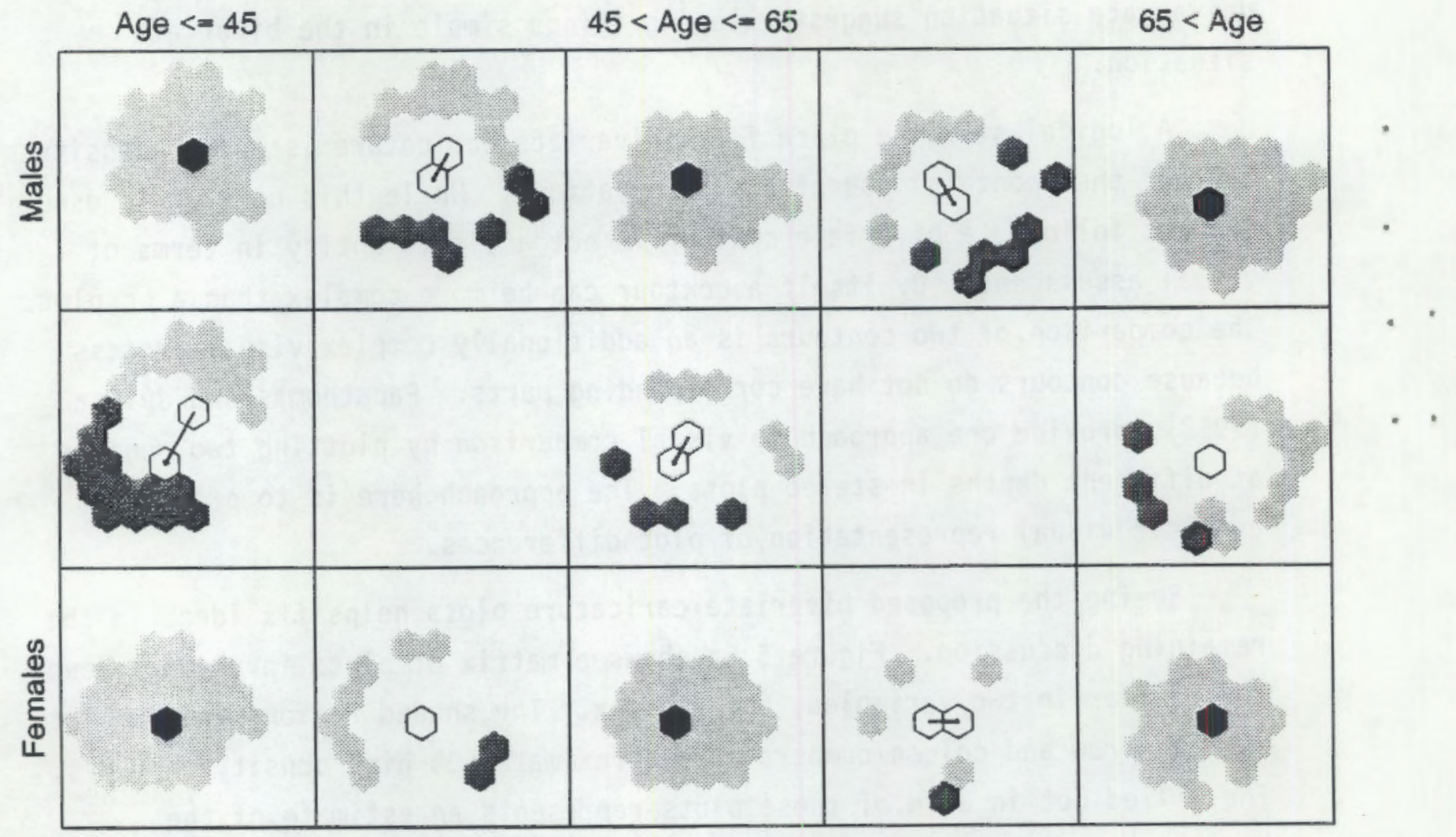

FIGURE 5.6a. A Density Caricatures and Comparison Plot Matrix. The data set comes for the NHANES I study. The variables, as indicated in $5.6 \mathrm{~b}$, are serum albumen and transferrin saturation. Plots with odd row and column numbers are density caricatures showing a $50 \%$ density contour in gray and an estimated bivariate median as a black hexagon. Plots with even row or column numbers are difference plots. The light shaded regions show the areas left behind in moving from the left (top) contour plot to the right (bottom) contour plot. The dark shaded region shows the area added in the transition. The outlined cells show the cell centers and the arrow shows the movement of the cell centers with the arrowhead pointing at the right (bottom) cell center. Note that as age increases the males distribution becomes similar to that of the females.

decrease with age until, in the over 65 age group, males and females are similar. The change in transferrin saturation is less marked. Males and females end up being similar, as the females values increase slightly. The contours look roughly elliptical and the median cells are well centered. 


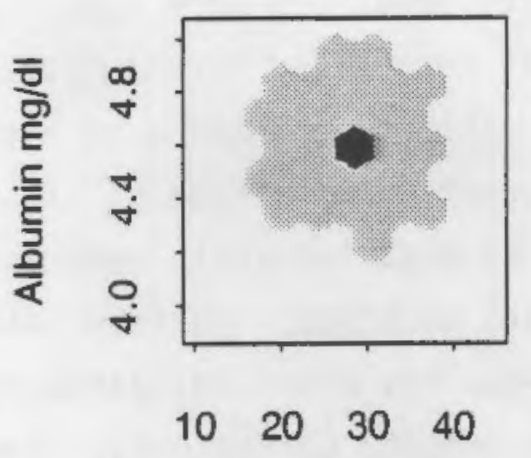

Transferrin Sat. (\%)

FIGURE 5.6b. A Plot Showing Scales and Axis Labels. This plot shows the axis labels and scales that are common to all plots in Figure 5.6a.

Interpretation involves issues of biological and statistical significance. Changes of a single plot cell may not be of much practical significance because such can be related to binning artifacts. Given the coarseness of the binning and a scale covering the $50 \%$ contours, differences of two cell widths are likely to be of biological significance. Statistical assessment of differences can be approached through comparison of individual cell densities (Carr, Littlefield, Nicholson, and Littlefield 1987) or through global assessment using nonparametric density estimates and likelihood ratio tests.

The design of Figure $5.6 \mathrm{a}$ is of greater importance than the construction details. However, a description of the construction details shows the simplicity of the current methods and may lead to development of better ones. With binned data, obtaining an approximate $50 \%$ high density contour is easy. The bin cells are sorted by cell counts and a cut off obtained using cumulative counts. The contour is obtained by plotting cell symbols that fill the binning region for each of high count cells above the cut off. With large data sets, the contour should be relatively stable but binning can introduce some artifacts. Smoothing the binned data helps produce more stable contours. 
In Figure 5.6a, two density smoothers were used. The first pass used a simple seven-hexagon-cell integer valued kernel that smoothes between adjacent cells, empty or not. The second pass used variable weights to restrict the smoothing to adjacent occupied cells. In effect, observations contribute to counts in cells at most two cells away and only one cell away in directions where no data occurred. An interesting question concerns the frequency with which high-quality cross-validated density estimates would lead to more informative density caricatures. For caricatures it might even be helpful to simplify the appearance of contours using a closing operation as described by Serra (1982).

In the univariate case, typical statements concern changes in the center of the distribution and changes in the distributional shape about the centers. By analogy, portraying some center for bivariate distributions is important. Small (1989) describes multivariate generalizations to the univariate median that might be used as the definition of center. All generalizations seem to have drawbacks, such as lack of affine invariance, breakdown in the presence of contaminating observations or computational intractability. In particular two of the more conceptually appealing approaches for defining observation depth (greatest depth from the "outside" corresponds to the median), Tukey's (1975) half space depth and Liu's (1988) simplicial depth, are not computationally tractable. For such methods approximations might be developed by considering case weighted cells in a coarse lattice. In lieu of such approximations, the approach here borrows from the image processing literature and determines centers by the process of cell erosion.

The solid black cells in Figure 5.6 a are centers determined by cell erosion. The erosion process is conceptualiy simple. The process starts with the cells in the contour. Counts are then repeatedly removed from the cells in proportion to the number of exposed faces ( 1 to 6 ) and cells with non-positive counts are removed. Each time a cell is removed the number of exposed faces of each of its contour neighbors is updated. The last cell removed is defined as the median. With large data sets, a single cell is usually eroded last. If more that one cell is removed at the last 
erosion cycle, a task specific final determination can be based on the cells tied at the least negative count.

Using erosion to select an existing hexagon cell as center is invariant under 60 degree rotations of the data. However the binning-erosion approach is not affine invariant so more complex approaches might be tried. One approach is to pool all subsets to be compared into a single set. Then the data set can be transformed into a canonical form before binning. If the pooled data set had a roughly elliptical distribution, Tyler's (1987) robust method for determining the mean and covariance might be used as the basis for such a transformation. Determination of the multivariate median proceeds as above. Then the inverse transformation is used to located the center cell in the binned plots based on the original data.

The last issue in the construction of Figure $5.6 \mathrm{a}$ is common treatment of subsets. The difference plots require the binning lattices to be identical. This is easily accomplished by using pooled data extrema in establishing the lattice point separations. Figure $5.6 \mathrm{a}$ zooms in on the contours. After combining the $50 \%$ contours to find the new boundaries, boundaries for one axis have to be extended to maintain the aspect ratio in data units. Otherwise the original hexagon cells will not plot as hexagons.

Generalization of Figure 5.6a can take several directions. For animation purposes some rearrangement of plots may be helpful. For example, if only the males were to be shown and there were many age categories, the difference plots might form a separate horizonal sequence below the juxtaposed contour plots. As for the content of each plot, keeping things simple requires discipline. When time is available to study images, many analysts would add some representation for the modes. In the hexagon context, one possibility is to plot the contour in gray and to represent modes using black hexagons whose size is a monotone function of the counts. A next step would be to look at the low density region. Examples of looking at low density regions are given in the following section. 



\subsection{COGNOSTICS FOR COMPUTER BASED SCREENING OF PDE SOLUTION SETS}

The term cognostics was coined by John Tukey (1982) to stand for computer guiding diagnostics. The notion of cognostics, computing diagnostics and using them in a computer-based inference procedure, is broad in scope and could cover many applications, especially in the area of expert systems. So far the primary accounts of cognostics (Tukey and Tukey 1985 and Tukey(a)) focus on prioritizing a large number of plots for human review. The large number of plots arise from picking variable pairs from a large number of "statistical" variables. The diagnostics are measures of interest based on plot features. The inference procedure is the trivial one of ranking plots based on the measures of interest. The examination process typically includes not only the most interesting of the plots but also some of the "least interesting" plots as part of visual calibration and algorithm verification.

Cognostics can be applied to many data bases. The early applications focused on observational data. However, the approach is equally applicable to computer generated output of Monte Carlo studies. Computer output needs to be examined, both to screen for inconsistencies and to scan for patterns that lead to new insight. Further, the cognostics concept can be extended to data bases of variables that are not routinely considered as having stochastic components.

The following examples are extracted from research in developing cognostics to assist in examining PDE (partial differential equations) solution sets. The examples, which are taken from computation fluid dynamics problems, have much structure and ' $n 0^{\prime}$ error so may seem foreign to statistical analysts. The following subsection provides background on such solution sets, and illustrates efforts to recast problems so that common statistical concepts and tools are applicable.

(a) Tukey, P. A. 1990. "Cognostics For Screening Multidimensional Data." Computational Statistics and Graphics - IMA Volumes in Mathematics and Its Applications. Springer-Verlag, New York. In press. 


\subsection{BACKGROUND ON PDE SOLUTIONS SETS}

Computational fluid dynamics solutions sets are generated as the solution to partial differential equations with boundary conditions that define the problem of interest. PDE solution sets are discrete approximations and have space-time indices denoted $(x, y, t)$ for twodimensional and $(x, y, z, t)$ for three-dimensional problems. Insufficient space-time resolution results in inadequate approximations. Consequently researchers in computational fluid dynamics are continually pushing for more powerful supercomputers. Solutions sets can be very large. In fact it is atypical to store whole solutions sets.

The variables that are computed as part of the solution set depend on the problem. Common variables include temperature, pressure, velocity, and vorticity but many more may be generated. The locations for scalar quantities such as temperature may differ from those of vector quantities, such as velocity. Scalars are often located at the center of 2-D or 3-D cells and vector quantities are located on cell faces. Averaging is often needed so that scalars and vectors correspond to the same locations and the solution at a particular location can be thought of as a multivariate observation.

The examination of computational fluid dynamics solution sets is something of an art form. In pre-workstation days, modelers would ignore reams of printed output to check the value of a few numbers in detail. Now that high-powered graphics workstations are available, animation viewing is common practice. Despite this tremendous step forward, (and ignoring simple tests built into the software) most portions of solutions sets go unexamined. One common animation format uses pseudo-color contours to represent values of one variable at nodes located in a 2-D slice of a 3-D model for a limited time period. The reasons for making the particular movie may be excellent, but surely argument can be made for screening of other aspects of the solutions sets.

An objective function is required to prioritize portions of the solution set for examination. If the objective function is to be based on 
internal consistency arguments and distributional assessment, the notion of replicates must be introduced. Since PDE solutions sets have unique spacetime indices for each multivariate observation, the notion of replicates is introduced in one of three ways: 1) by considering "replicate" solutions sets that differ only in their spatial resolution, 2) by considering symmetry in solution sets, and 3) by ignoring selected space-time indices. Approach 1) is common to PDE modelers because the adequacy of mathematical and numerical approximations to the equations is known to be resolution dependent. Approach 2) is uncommon because additional resolution can be obtained assuming that symmetric answers are identical and not computing them. Approach 3) is quite rare since it drops fundamental parts of the defining equations.

While cognostics have been developed to assess symmetry departures, the following examples are based on approach 3 ). That is, the presumption is made that low density patterns produced by ignoring indices can call attention to infrequent events that are of interest and that density patterns may provide new insights. Readers are warned that the simple approach of dropping indices may not be a productive one. More needs to be learned about the conditions under which the approach is useful. For example, dropping the time index may be most productive when there is an attractor in the state space. Perhaps more elaborate aggregation approaches will be required. For example Kim and Moin (1986) use a vorticity detector to identify conditions under which data can be aggregated to help determine the shape of conjectured horse shoe vortices.

Cognostics provide a way of screening PDE solutions sets for interesting or suspect patterns. However, the transition from this general idea to fruitful application is non-trivial. Some patterns catch the eye because of their graphic design and the operations of the eye-brain system. Such eye catching patterns may not have useful interpretations. Some eye catching patterns have generic interpretive interest to statistical analysts. Figure 6.1 shows an ordinary scatterplot matrix from a single 


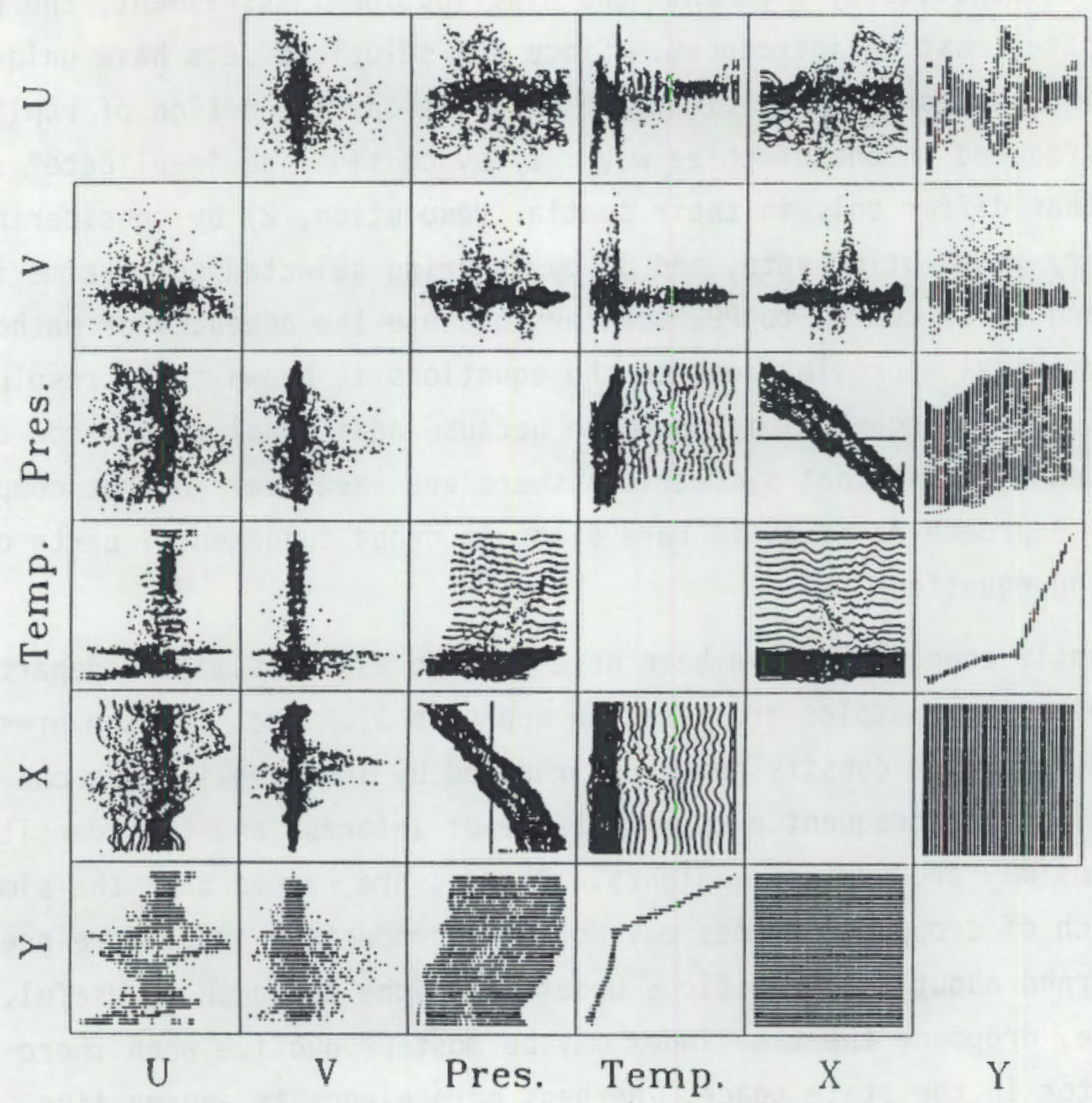

FIGURE 6.1. A Scatterplot Matrix. This standard scatterplot shows data from time step 49 of a low resolution 2-D PDE Thundercloud model. The variables $u$ and $v$ are velocity components in centimeters per second. Pressure refers to perturbation pressure (non-dimensionalized difference of pressure from the base state). Temperature refers to potential temperature $(K)$. The variables $x$ and $y$ are spatial components, horizontal position and altitude above ground, respectively. Several patterns appear that are of generic interest. Examples include the functional relationship between temperature and $y$ (altitude) with the jog at the boundary layer and the sudden change in variability of $v$ as a function of temperature. 


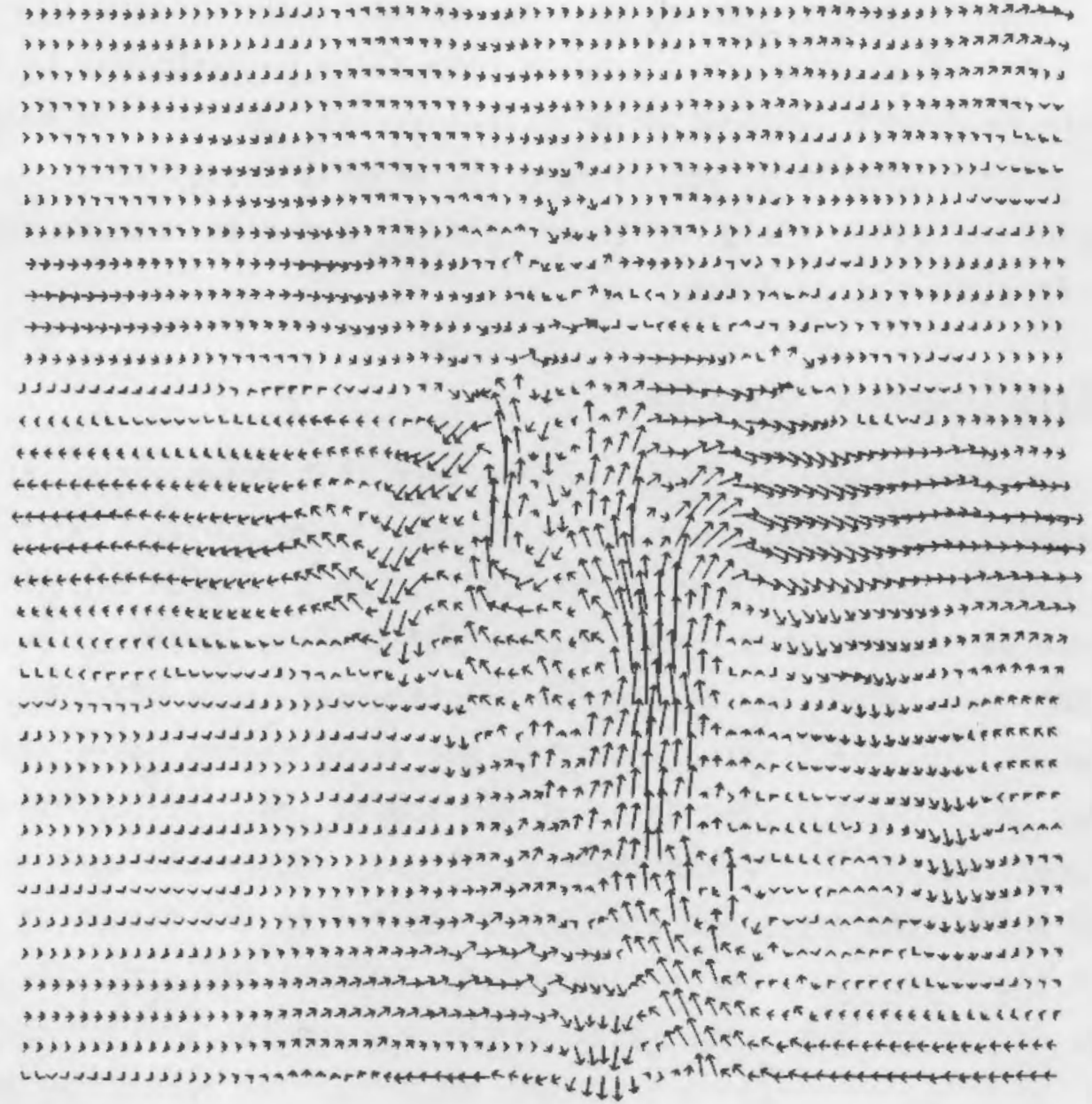

FIGURE 6.2. A Velocity Vector Plot. This 2-D velocity vector plot shows a cross section of a PDE Thundercloud simulation. The vertical axis is altitude above ground and the grid spacing between vectors represents 500 meters in both directions. Note the downward pointing vectors near the bottom center of the plot. Such regions make for hazardous flying.

time step of a very low resolution 2-D thundercloud model (Cotton, Stephens, Nehrkorn, and Tripoli 1982). Generic patterns of interest include a strong functional relationship in the temperature versus $y$ (altitude) plot and the sudden change in variability in the temperature versus $u$ and $v$ components of velocity plots. Some patterns require the subject matter context to be recognized as important. Figure 6.2 shows velocity vectors from a cross section of the thundercloud. The regions 
where the velocity vectors point down are of particular interest in the context of flying an aircraft. Finally, important patterns may be difficult for the eye to detect (without major graphics redesign) but are readily detected using algorithms. For example the eigenvalues of local Jacobians of the velocity vector field can be of interest but difficult to assess visually from the vector field.

\subsection{DENSITY BASED ARM DETECTION}

In examining bivariate plots, one pattern that draws visual attention is low density arms radiating out of high density regions. Figure 6.3 shows an example (see also Carr et al. 1987). This hexagon bin plot of temperature versus pressure comes from a TEMPEST (see Trent, Eyler and Budden 1985) generated, low resolution solution set for a glass melter simulation model. The observations from all fluid region nodes and 100 time steps are included. The spike at the top of the plot represents unusual pressure values. After checking, it was determined that the values came from a few time steps and from nodes located in the trough at the bottom of the melter as show in Figure 6.4. While this particular finding triggered no new insights, the spike at the top and the arms on the top left are the kinds of patterns that have potential for generating new insight.

Figure 6.3 motives the following arm detection algorithm. The high count cells containing $96 \%$ of the data are excluded from consideration as arms. Then a thinning process (Rosenfeld and Kak 1982) removes selected low density cells. Thinning is much like the erosion described above except the cells are treated as being present or absent and only selected cells are eroded. In particular, cells are not eroded if they have only one neighbor or if their removal would create a locally disconnected cells. The thinning process produces skeletal structure as shown in Figure 6.5. The measure of interest for such a plot is defined are the number of cells in the skeleton divided by the number of cells subject to erosion. 


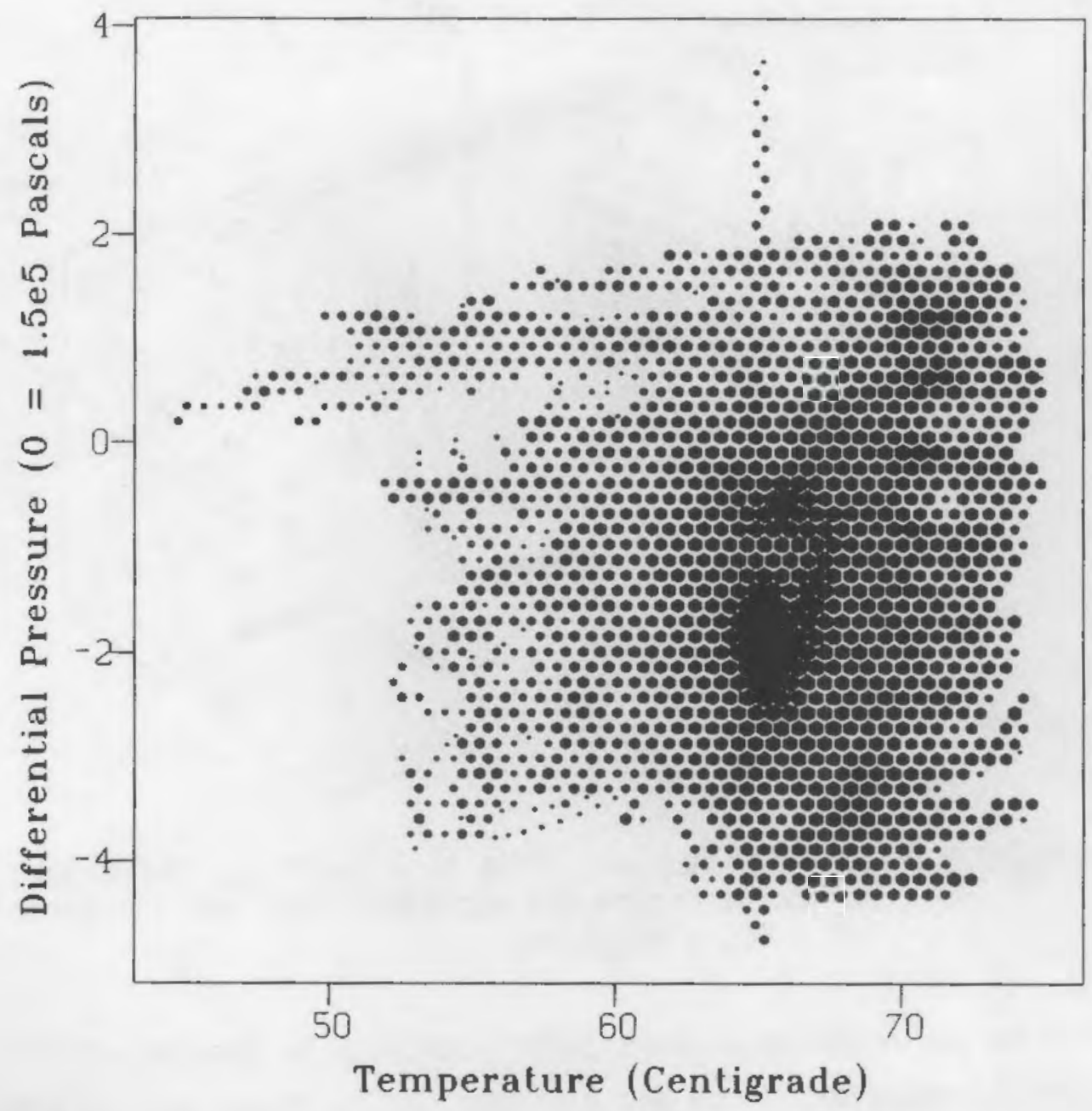

FIGURE 6.3. A Hexagon Bin Plot. The plot represents the temperature and pressure values of 243,600 space-time nodes in a PDE glass melter simulation. Hexagon area is linearly related to the $\log$ base 10 of the counts. The spike of unusual pressure values at the top of the plot draws attention. The values come from node located in the melter trough as shown in Figure 6.4.

Like other heuristic cognostics, this algorithm can pick up unexpected patterns and may not pick up all the desired ones. As part of the evaluation process the algorithm was turned loose on pairs of thirteen variables in the glass melter solution set. The algorithm picked out the plot in Figure 6.6 as having the highest armness measure of the 78 plots. The loops in Figure 6.6 are intriguing. Likely the loops could be made to 


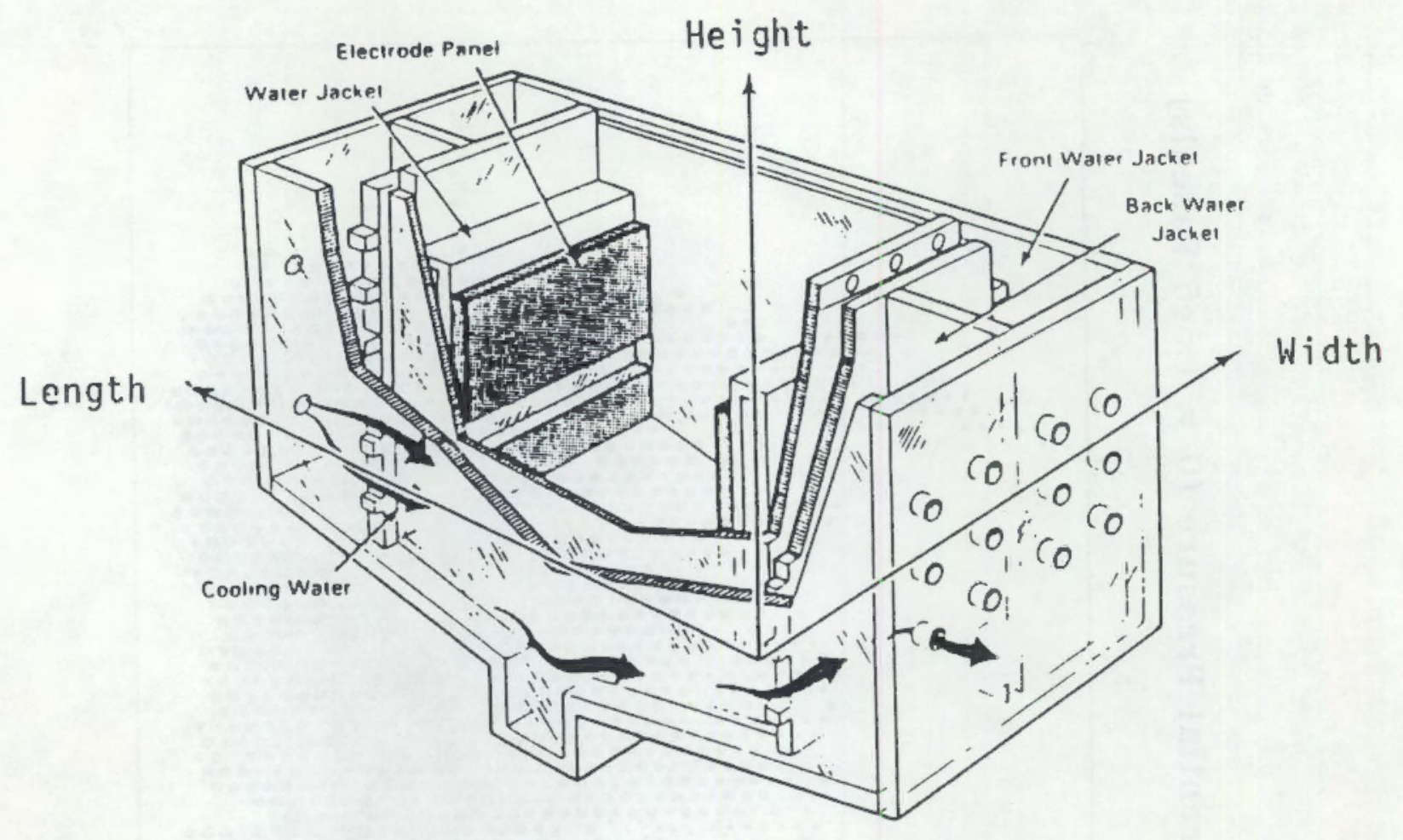

FIGURE 6.4. A Glass Melter. This is a sketch of the glass melter experimental apparatus that was the basis of a PDE simulation.

disappear by adding nodes at particular locations in the low resolution melter model. However, loops and clusters can represent unique phenomena. For example, in atmospheric studies velocity vectors associated with the jet stream will be distinctive even though nodes are added near its boundary.

Consideration of velocity and vorticity vectors for three-dimensional PDE solutions sets motivates generalizing the armness measure to threedimensional binned plots. The major consideration in this generalization is selection of a thinning algorithm for truncated octahedron binned data. The particular algorithm developed here utilizes both cell counts and exposed surface area of the cells to determine the order in which cells are visited in the thinning process. Figure 6.7 shows the skeletal structure for the low density $5 \%$ of the data when the algorithm is applied to velocity data. The velocity data is from time step 151 of a model of 


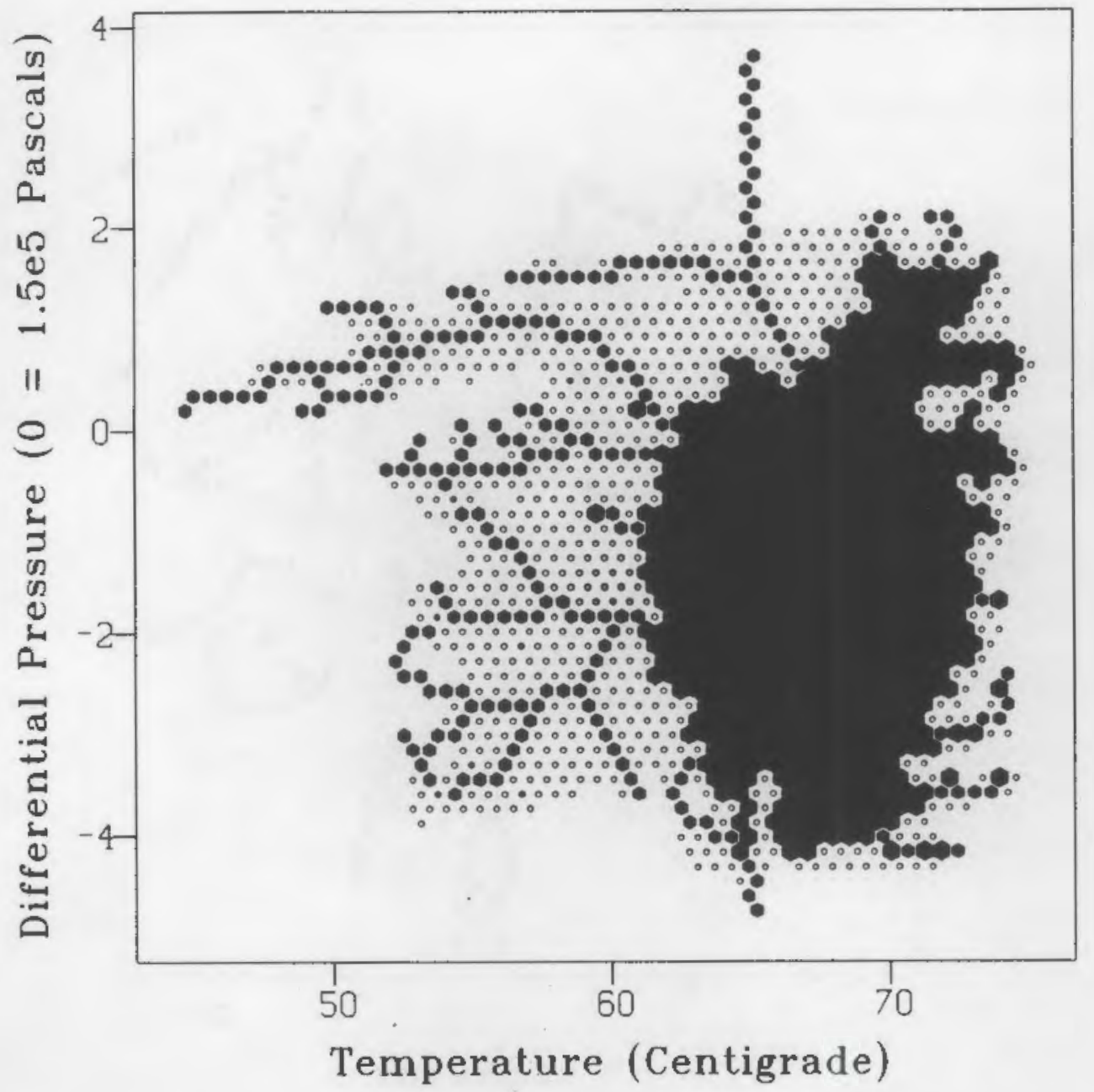

FIGURE 6.5. A Skeleton Plot. This plot shows the result of applying a thinning based arms detection algorithm. Cells with the largest counts that include $96 \%$ of the data were protected from the erosion (thinning) process. The solid black region in the center of the plot represents protected cells. Small open hexagon represent eroded cells. Small filled hexagons show isolated cells and one cell holes which are filled before thinning. Filled hexagons that almost fill the binning regions show the skeletal (arm) structure. While perhaps more cells should be protected from thinning, the algorithm finds the intended spike at the top of the plot and the long arm on the upper left. The measure of interest for the plot, 0.30, is defined as the number of skeletal cells divide by those subject to erosion. 


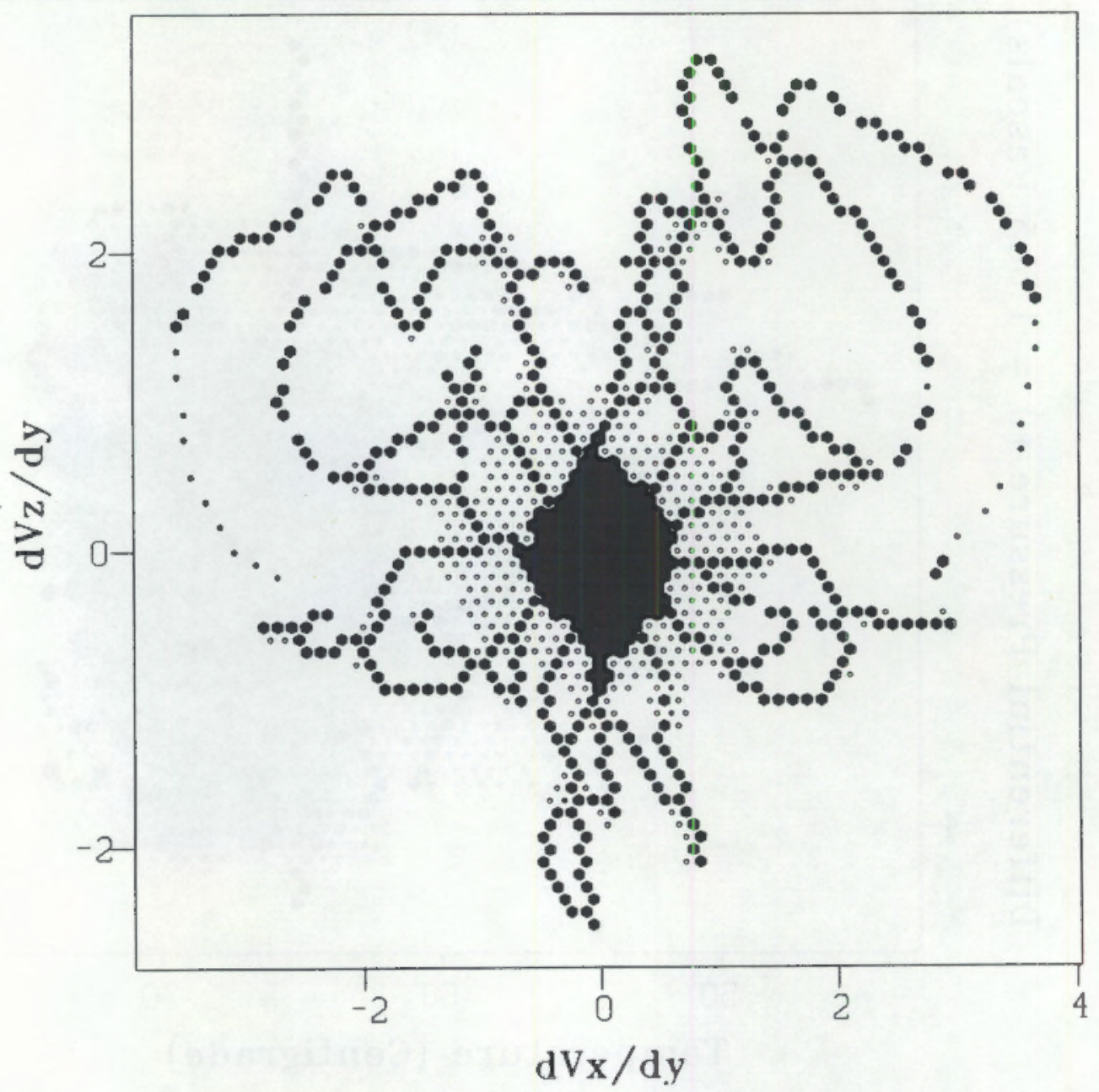

FIGURE 6.6. An Extreme Skeleton Plot. An arms detection cognostic was applied to binned bivariate plots of 13 variables in a PDE glass melter solution set. This particular plot, which involves spatial derivatives of velocity components, was the most extreme case with armness measure of D.61. Given such plots with arms, the next step is to identify the corresponding nodes in the glass melter model.

axial flow in a square duct with inlet and outlet boundary conditions. In contrast to Figure 6.6 , the plot suggests fuzz around the high density region and the armness measure of 0.14 seems low. Making a stronger statement about the 3-D measure requires further experience or a reference distribution for comparison. 

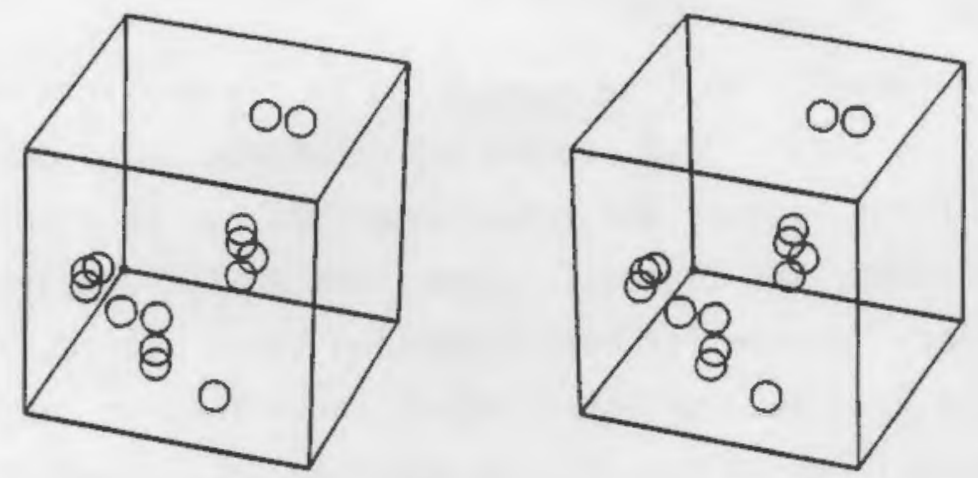

FIGURE 6.7. A Stereo Pairs 3-D Skeleton Plot. Like Figure 5.4, the images can be fused without the assistance of a stereoptican. A small square dot on the plot frame marks the corner furthest from the viewer.

The three variables are PDE solution set velocity components from a simulation of axial flow in a square duct. The 8000 observations for one time step are binned into truncated octahedron regions. Open circles represent skeletal cells produced by thinning the low count cells containing $5 \%$ of the observations. The armness measure, 0.14 , increased to 0.19 when cells containing $50 \%$ of the counts were subject to thinning. In either case this is small compared to the armness measure for Figure 6.6. Here the arms are more like fuzz attached to the high density region.

Methods for three dimensions deserve more study. Alternative choices for a thinning algorithm change the position of parts of the skeletal structure. The order of visiting the cells determines whether or not someskeletal cells are inside or outside the protected region and this affects the armness measure. Alternate algorithms and definitions for the armness measure are worthy of further study. Such study should address the sensitivity of the measures to changes in bin resolution. In addition, displays of 3-D structure will help provide insight. Variations on the display of skeletal structure are possible. Rather than plotting circles as approximate cross sections of truncated octahedrons as in Figure 6.7 , lines can be drawn between the centers of adjacent skeletal cells. Less jagged representations of the skeletal structure can then be developed by generalizing the work of Sirjani and Cross (1989). Opportunities for further research in density based arm detection are numerous. 


\subsection{PRACTICAL CONSIDERATIONS}

Practical aspects must be considered in the application of cognostics to PDE solutions sets. Post-processing of stored solutions has three advantages. First, scales and transformations can be determined using hindsight. Second, the cognostic algorithms do not appear to compete with the PDE modelers' desire for more computing time. Third, after high interest plots have been selected deeper investigation can be pursued by making additional passes through the solution set. However, post-processing is often impractical due to the storage/access problems for huge solutions sets. To address the storage/access problem, a variety of cognostics were implemented to run in parallel on an Encore Multimax Parallel Processor. This allows the cognostics to be computed for one time step while TEMPEST, a flow solver code, is generating the solution set for the next time step. As opposed to the above temporal overview examples, the parallel cognostics are computed at each time step and the binned data for high interest 2-D and 3-D plots are stored for later examination.

Timings for the axial flow model mentioned in Section 6.2 show that the cognostics implemented to date run much faster than the flow solver code. This includes the 3-D arms detection algorithm. Consequently most of the processors can be devoted to the flow solver code. The goal is to make the cognostics as productive and painless as possible.

Since the evaluation of cognostics is in progress, the ability to experiment is important. The TEMPEST/cognostics interface provides access to a variety of cell centered scalars and vectors and allows selected algorithms to be applied to selected variable pairs or triples without recompiling. The interface also allows cell residuals from local quadratic models to be included as variables and incorporates appropriate sign changes for symmetric models. In TEMPEST, the node placement is fixed from time step to time step. The local residual computation is performed rapidly by precomputing and storing the coefficients for the linear combinations of dependent variables that yield residuals. 
The linking of plots (Stuetzle 1987) is a useful tool in the evaluation process. When an unusual density pattern appears, the PDE modeler wants to know the location of the nodes involved. Thus the mapping from binning regions to nodes must be saved along with the high interest plots.

Practical problems remain. Scaling on the fly complicates binned comparison over time. Transformations can be important in obtaining decent resolution in the binned data, but many not be obvious in advance. Finally, experience is required (or some kind of learning algorithm) to pick cognostic cut offs that save just the most interesting plots. These typical problems will recur as data generation/collection rates force more emphasis on real time processing. 


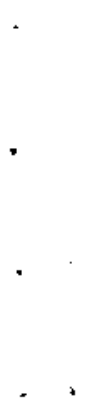




\subsection{CLOSING REMARKS}

The algorithms for the above graphics and cognostics are simple. By the time this report is published, most of them should be available to the New S (Becker, Chambers, and Wilks 1988) users group over national networks. Variants of the algorithms should be helpful in looking at yesterday's large data sets.

The prospect of thoroughly exploring tomorrow's large data sets is less than bleak. The data generation/collection rates dictate that almost all looking will have to be done by algorithm. Much research is needed to integrate the tools of computational statistics, image processing, and human perception, so that large data sets can be scanned for general features. Such research can lay the foundation for initial supervised training of neural networks and the development of more advanced real time algorithms.

Large data set analysis depends on politics as well as methodology. Politics determine what funding is available for analysis. A major bottleneck is the small amount of money allocated for data analysis relative to that allocated for collection or generation. The open minded flexibility of exploratory analysis costs money. Exploratory analysis can be hard to sell since the results are not specified in advance and may raise questions about accuracy and sampling characteristics of the observations. Data analysts will continue to have an up hill educational battle to include exploratory/robust methods whenever the additional analys is is either labor or computationally intensive.

Scientific freedom issues are involved in access to data sets. Access includes having the right hardware, software, documentation, and permission. The tentacles of the intellectual property movement will undoubtedly extend to data sets. Thus attempting to look at large data sets provides challenges in both technical and political arenas. 


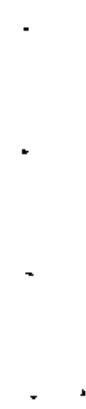




\subsection{ACKNOWLEDGMENTS}

The work described in this report is based on the contributions of many people. People who contributed data or solution sets that were used either directly or in background work include Richard G. Stevens, Loren L. Eyler, David C. Bader, and James R. GT imm. People who contributed to the development and implementation of cognostics for PDE solution sets include Wesley L. Nicholson, D. Richard Iltis, Patrick S. Lowery, David M. Elwood, Kristine B. Sauer, and Nancy E. Miller. Valuable comments were received from IMA workshop participants. 
- 


\subsection{REFERENCES}

Becker, R. A., J. M. Chambers, and A. R. Wilks. 1988. The New S Language. Wadsworth and Brooks/Cole, Pacific Grove, California.

Bertin, J. 1983. Semiology of Graphics. Translated by W. J. Berg. The University of Wisconsin Press, Madison, Wisconsin.

Carr, D. B., W. L. Nicholson, R. J. Littlefield, and D. L. Hall. 1986. "Interactive Color Display Methods for Multivariate Data." Statistical Image Processing and Graphics, eds. E. J. Wegman and E. J. Depriest, Pp. 215-250. Marcel Dekker, New York.

Carr, D. B., R. J. Littlefield, W. L. Nicholson, and J. S. Littlefield. 1987. "Scatterplot Matrix Techniques for Large N." Journal of the American Statistical Association 82:424-4436.

Cleveland, W. S., and S. J. Devlin. 1988. "Locally Weighted Regression: An Approach to Regression Analysis by Local Fitting." Journal of the American Statistical Association 83(403):596-610

Cleveland, W. S., and R. McGill. 1984a. "Graphical Perception: Theory, Experimentation, and Application to the Development of Graphics Methods." Journal of the American Statistical Association 79:531-554.

Cleveland, W. S., and R. McGill. 1984b. "The Many Faces of a Scatterplot." Journal of the American Statistical Association 79:807-822.

Cotton, W. R., M. A. Stephens, T. Nehrkorn, and G. J. Tripoli. 1982. "The Colorado State University Three-Oimensional Cloud/Mesoscale Mode1-1982: Part 2: An Ice Phase Parameterization." Journal De Recherches Atmospheriques 16:295-320.

Conway, J. H., and N.J.A. Sloane. 1982a. "Voronoi Regions of Lattices, Second Moments of Polytopes and Quantization." IEEE Transactions on Information Theory IT-28(2):211-226

Conway, J. H., and N.J.A. Sloane. 1982b. "Fast Quantizing and Decoding Algorithms for Lattice Quantizers and Codes." IEEE Transactions on Information Theory IT-28(2):227-231

Dekinder, R. E. Jr, , G, G. BTackman, and L. Gibson. 1982. "Application of an N-Dimensional Algebra to Processing 'Battle Field Obscuration' StereoPair Video Data." Processing and Display of Three-Dimensional Data, Proceedings of SPIE - The International Society for optical Engineering, ed. J. J. Person, Vol, 367, pp. 57-67.

Fabbri, A. G. 1984. Image Processing of Geological Data. Van Nostrand Reinhold, New York. 
Friedman, J. H. 1984. A Variable Span Smoother. Technical Report LC55, Dept. of Statistics, Stanford University, Stanford, California.

Frigge, M., D. C. Hoaglin, and B. Iglewicz. 1989. "Some Implementations of the Boxplot." The American Statistician (43) 1:50-54.

Hahn, G. J. 1989. "Statistics-Aided Manufacturing: A Look into the Future." The American Statistician (43)2:74-79.

Julesz, B. and J. R. Bergen. 1983. "Textons, The Fundamental Elements in Preattentive Vision and Perception of Textures." The Bell System Technical Journal (Human Factors and Behavioral Science) 62(6):1619-1645.

Kim, J., and P. Moin. 1986. "The Structure of Vorticity Fields in Turbulent Channel Flow. Part 2. Study of Ensemble-Averaged Fields." J. Fluid Mechanics 162:339-363.

Liu, R. Y. 1988. "On a Notion of Simplicial Depth." Proceedings of the National Academy of Science. USA 85, pp. 1732-1734.

Papathomas, T. V. and B. Julesz. 1988. "The Application of Depth Separation to the Display of Large Data Sets." Dynamic Graphics For Statistics, eds. W. S. Cleveland and M. E. McGill, pp. 353-377. Wadsworth and Brooks/Cole. Belmont, California.

Riggan, W. B., J. Van Bruggen, J. F. Acuavella, J. Beaubier, and T. J. Mason. 1983. U.S. Cancer Mortality Rates and Trends, 1950-1979. Volume 2. U.S. Environmental Protection Agency and the National Cancer Institute, Washington, DC.

Rosenfe1d, A., and A. C. Kak. 1982. Digital Picture Processing. Volume 2, Second Edition. Academic Press, New York.

Scott, D. W., and G. R. Terrel. 1986. Biased and Unbiased Cross-Validation in Density Estimation. Technical Report No. 23, Department of Statistics, Stanford University, Stanford California.

Scott, D. W. 1985. A Note on Choice of Bivariate Histogram Bin Shape. Technical Report 85-311-3, Dept. of Mathematical Sciences, Rice University, Houston.

Serra, J. 1982. Image Analys is and Mathematical Morphology. Academic Press, New York.

Sirjani, A., and G. R. Cross. 1989. On Representation of a Shape's Skeleton. Technical Report CS-89-203, Computer Science Department, Washington State University, Pullman, Washington. 
Sma11, C. G. 1989. A Survey of Multidimensional Medians. Statistics

Technical Report Series Stat-89-08, Department of Statistics and Actuarial Science, University of Waterloo, Waterloo, Canada.

Stuetzle, W. 1987. "Plot Windows." Journal of the American Statistical Association 82:466-475.

Trent, D. S., L. L. Eyler, and M. J. Budden. 1985. A Three-Dimensional Time-dependent Computer program for Hydrothermal Analys is. PNL-4348, Pacific Northwest Laboratory, Richland, Washington.

Tukey, J. W. 1975. "Mathematics and the Picturing of Data." Proceedings of the International Congress of Mathematicians, Vol. 2, 523-531. Vancouver, B. C., 1974.

Tukey, J. W. 1982. "Another Look at the Future." Computer Science and Statistics: Proceedings of the 14th Symposium on the Interface. SpringerVerlag, New York.

Tukey, J. W. 1989. "Data Based Graphics: Visual Display in the Decades to Come." Proceedings of the American Statistical Association Sesquicentennial Invited Papers. American Statistical Association. Alexandria, Virginia.

Tukey, J. W. and P. A. Tukey. 1985. "Computer Graphics and Exploratory Data Analysis: An Introduction." Proceedings of the 6th Annual Conference and Exposition, Vol. 3, pp. 773-785. National Computer Graphics Association, ISBN 0-941514-0909.

Tyler, D. E. 1987. "A Distribution-Free M-Estimator of Multivariate Scatter." Annals of Statistics 15:234-251.

Wahba, G. 1986. "Multivariate Thin Plate Spine Smoothing with Positivity and Other Linear Inequality Constraints." Statistical Image Processing and Graphics, eds. E. J. Wegman and D. J. Depriest, Pp. 275-289. Marcel Dekker, New York.

Wainer, H. and C. M. Francolini. 1980. "An Empirical Inquiry Concerning Human Understanding of Two-VariabTe Color Maps." The American Statistician $34(2): 81-93$. 
.

. 


\section{DISTRIBUTION}

No. of

Copies

OFFSITE

D. M. Austin

U.S. Department of Energy

Division of Scientific

Computing, ER-7

Office of Energy Research

Washington, OC 20545

12 DOE Office of Scientific and Technical Information

\section{R. A. Becker}

AT\&T Bell Laboratories 600 Mountain Avenue

Murray Hill, NJ 07974

R. Beckman

Los Alamos National Laboratory

P.0. Box 1663, MS F600

Los Alamos, NM 87545

J. Caberra

Department of Statistics

Rutgers University

Hill Center, Busch Campus

New Brunswick, NJ 08903

W. S. Cleveland

AT\&T Bell Laboratories

600 Mountain Hill Avenue

Murray Hill, NJ 07974
W. F. Eddy
Department of Statistics
Carnegie-Mellon University
Pittsburgh, PA 15213
C. R. Goodall
Princeton University
ACE 42, Engineering Quadrangle
Princeton, NJ 08544

No. of

Copies

H. Chernoff

Department of Statistics

Harvard University

Cambridge, MA 02138

J. B. Gray

M.J. Neeley School of Business

Texas Christian Univiversity

Box 32868

Fort worth, TX 76129

M. Johnson

The Upjohn Company

301 Henrietta Street

Kalamazoo, MI 49001

S. Keller-McNulty

Department of Statistics

Kansas State University

Manhattan, XS 66506

J. A. MCDonald

Department of Statistics

University of Washington

Seattle, WA 98195

A. McIntosh

Bel1 Communications Research

445 South Street

Morristown, NJ 07960-1910

J. 0. Pedersen

Xerox Palo Alto Research Center

3333 Coyote Hill Road

Palo Alto, CA 94304

D. Pregibon

AT\&T Bell Laboratories 600 Mountain Avenue

Murray Hill, NJ 07974 
No. of

Copies

D. W. Scott

Department of Statistics

Rice University

P.0. Box 1892

Houston, TX 77251-1892

W. Stuetzle

Department of Statistics

University of Washington

Seattle, WA 98195

M. Thoma

BMDP

1440 Sepulveda

Los Angeles, CA 90025

R. A. Thisted

Department of Statistics

University of Chicago

5734 University Avenue

Chicago, IL 60637

L. Tierney

School of Statistics

University of Minnesota

Minneapolis, MN 55455

E. R. Tufte

1161 Sperry Road

Cheshire, CT 06410

P. A. Tukey

Bell Communications Research

435 South Street

Morristown, NJ 07960

E. J. Wegman

George Mason University

242 Scjence Tech. Bldg.

Fairfax, VA 22030
No. of

Copies

L. Wilkinson

180 Sherman Avenue

Evanston, IL 60201-3715

A. R. Wilks

AT\&T Bell Laboratories

600 Mountain Avenue

Murray Hill, NJ 07974

\section{FOREIGN}

J. A. Nelder

Imperial College

Huxley Bldg., 180 Queens Gate

London, SW7 $2 \mathrm{BZ}$

United Kingdom

R. W. 01dford

Department of Statistics

University of Waterloo

Waterloo, Ontario, N2L 3G1

Canada

\section{ONSITE}

DOE Richland Operations Office

R. F. Christensen

Pacific Northwest Laboratory

DB Carr (26)

Publishing Coordination

Technical Report Files 


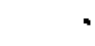

.

, 